\title{
The role of a proto-Schelde River in the genesis of the southwestern Netherlands, inferred from the Quaternary successions and fossils in Moriaanshoofd Borehole (Zeeland, the Netherlands)
}

\author{
A.A. Slupik ${ }^{1,}{ }^{*}$, F.P. Wesselingh ${ }^{2}$, D.F. Mayhew ${ }^{1,2, \dagger}$, A.C. Janse ${ }^{2}$, F.E. Dieleman ${ }^{2}$, \\ M. van Strydonck ${ }^{3}$, P. Kiden ${ }^{4}$, A.W. Burger ${ }^{5}$ \& J.W.F. Reumer ${ }^{1,6}$
}

1 Natuurhistorisch Museum Rotterdam, Westzeedijk 345, 3015 AA Rotterdam, the Netherlands

2 Naturalis Biodiversity Center, P.0. Box 9517, 2300 RA Leiden, the Netherlands

3 Koninklijk Instituut voor het Kunstpatrimonium, Jubelpark 1, 1000 Brussel, Belgium

4 TN0 - Geological Survey of the Netherlands, P.0. Box 80015, 3508 AL Utrecht, the Netherlands

5 P. Soutmanlaan 18, $1701 \mathrm{MC}$ Heerhugowaard, the Netherlands

6 Department of Geosciences, Utrecht University, P.0. Box 80021, 3508 TA Utrecht, the Netherlands

* Corresponding author. Email: slupik@planet.nl

$\dagger$ To our regret Dr David Mayhew passed away 3 October 2012. We would like to attribute this paper to the memory of this great scientist and colleague.

Manuscript received: June 2012, accepted: March 2013

\section{Abstract}

We investigated the Quaternary lithological succession and faunas in a borehole near Moriaanshoofd (Province of Zeeland, SW Netherlands), in order to improve our understanding of the depositional context of classical Gelasian mammal faunas from the region. The fossils mostly derive from the base of a fossil-rich interval between $31 \mathrm{~m}$ and $36.5 \mathrm{~m}$ below the surface, that was initially interpreted as a Middle or Late Pleistocene interglacial marine unit, but turned out to be a Late Quaternary fluvial unit with large amounts of reworked fossils and sediments. Eocene mollusc taxa pinpoint Flanders (Belgium) as the source region for this river. Within the base of this paleo-Schelde River fossil material of various stratigraphic provenance became incorporated.

Keywords: Pleistocene, Eocene, stratigraphy, reworking, Schelde, mammals, molluscs

\section{Introduction}

While studying stratigraphic successions in a borehole near the Schelphoek (Fig. 1; Slupik et al., 2007), uncertainties remained about the intervals overlying the Early Quaternary (Gelasian) Maassluis Formation. Deposits directly overlying the Maassluis Formation were previously tentatively assigned to the Eem Formation which is of the Eemian age (MIS 5e) (Slupik et al., 2007) with an assumption that these deposits could also originate from some periods within the Middle Pleistocene. Also the provenance of the mammal faunas from the adjacent Oosterschelde estuary (De Vos et al., 1995, 1998; Mayhew et al.,
2008; Reumer et al., 1998, 2005) remains uncertain. Since 1955, once a year a fishing-vessel expedition is organised with the aim to search for fossils at the bottom of two 40-45 $\mathrm{m}$ deep tidal channels in the Dosterschelde estuary (see for detailed information in Reumer et al., 1998, 2005). The fossils are dredged by mean of fishing-nets. Through the decennia of collecting activities, a large amount (hundreds of fossils) of mostly Early Pleistocene vertebrate fauna has been assembled and the composition of the fauna is thought to be well known. But the fossils are not available for direct observation in the sedimentary record, they are found ex situ, at a depth of 40-45 m below the water surface. Therefore their geological position within the 


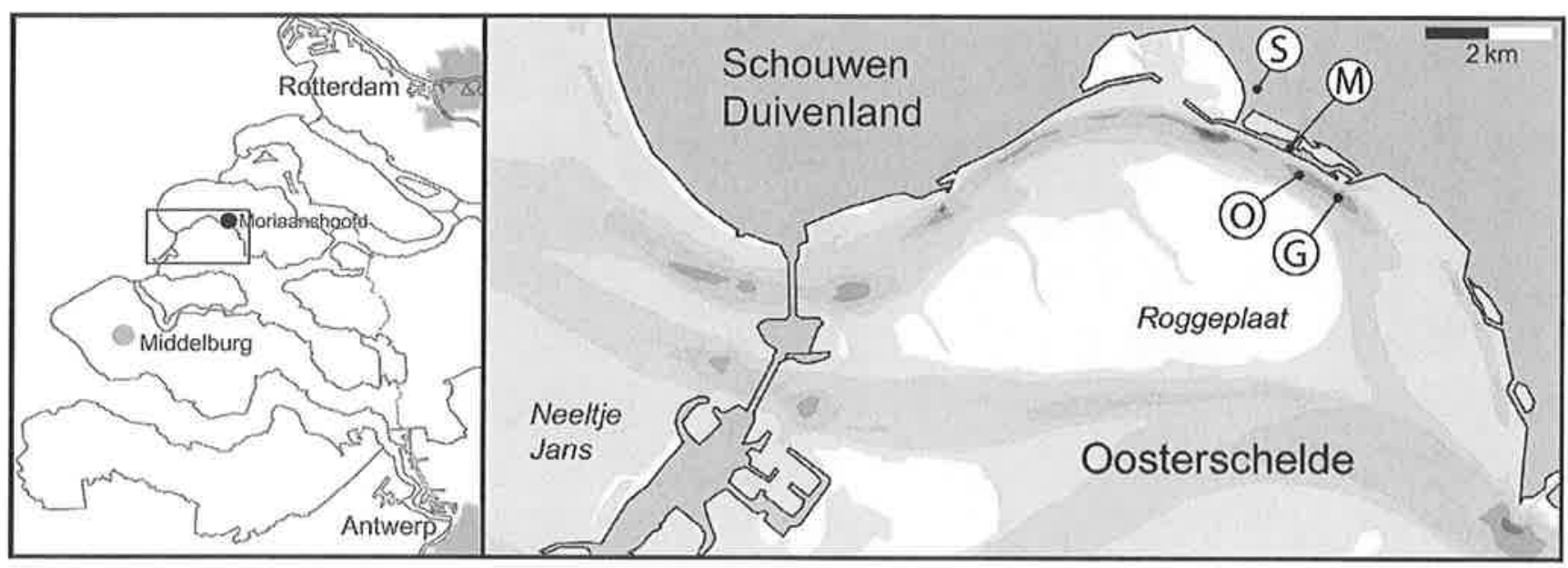

Fig. 1. Location of the borehole (with detailed map). Dredging localities: 0 - Olifantenputje; $G$ - Gastenputje; S-Schelphoek borehole; M-Moriaanshoofd borehole (this paper) (coordinates: $3^{\circ} 50^{\prime} 09^{\prime \prime} E, 51^{\circ} 40^{\prime} 50^{\prime \prime} \mathrm{N} ; \mathrm{RDX}=047.740 ; \mathrm{ROY}=411.380$ ).

succession remains unknown. It is not clear from which stratigraphic unit the 0osterschelde vertebrate fauna derives. The stratigraphic provenance of the fauna still has to be clarified.

In order to improve our understanding of the Quaternary successions in general and the depositional context of the mammal faunas of Gelasian age in particular we drilled a second borehole closer to the present-day tidal channels of the dredged fauna (De Vos et al., 1995, 1998; Mayhew et al., 2008; Reumer et al., 1998, 2005) (Fig. 1).

\section{Materials and methods}

The borehole is located south of Moriaanshoofd on the island of Schouwen-Duiveland (province of Zeeland; southwest Netherlands). A suction core was performed by the Van Elburg Drilling Company, between 25 and 28 august 2008 (Fig. 1). The borehole was located adjacent to the 0osterschelde dike south of Moriaanshoofd and reached an end depth of $62 \mathrm{~m}$ (TNO Geological Survey of the Netherlands borehole number B42G0769). The surface elevation at the borehole location was $4.19 \mathrm{~m}+$ N.A.P. (Dutch Ordnance Level, $\sim$ mean sea level).

Coring was performed in half-metre intervals. From each interval about half a kilogram of raw sediment was taken for lithological description and analyses. Additionally, larger samples of approximately $2-3 \mathrm{~kg}$ of sediment were taken at one-metre intervals and washed and sieved (sieve mesh $1 \mathrm{~mm}$ ) and analysed for fossil content (see procedures in Slupik et al., 2007). Fossils were picked from wash residues and identified. Molluscs were counted. All sediment samples were visually and microscopically examined. Lithological descriptions follow procedures outlined in Bosch (1999). Grain size was partly determined by visual sample description (using microscope Wild M5A equipped with ocular micro-scale measure bar) and partly performed on a laser particle sizer Malvern Instruments Ltd, UK at the Department of Earth Sciences, University of Utrecht.
The sediment samples and mammal remains of the Moriaanshoofd borehole are archived at the Natuurhistorisch Museum Rotterdam (NMR) and the molluscs are archived at the Naturalis Biodiversity Center in Leiden. Comparison of the colour of the sediments between the two boreholes proved difficult, as the analysis of the Moriaanshoofd samples took place within a year after drilling, whereas the Schelphoek samples were analysed after several decades, so that oxidation had affected the colour significantly.

Six radiocarbon datings on peat and wood fragments were performed by the laboratory of the 'Koninklijk Instituut voor het Kunstpatrimonium' (the Royal Institute for Cultural Heritage) in Brussels, Belgium, following procedures outlined in Van Strydonck and Van der Borg (1990-1991), and reported following Stuiver and Pollach (1977). All accelerator mass spectrometry (AMS) measurements follow Nadeau et al. (1998).

The lithostratigraphic terminology follows Weerts et al. (2000), Westerhoff et al. (2003) and TNO (2013) and the chronostratigraphy is according to the ICN/IUGS (2009) definitions. All depths are given below the ground surface (4.19 m+N.A.P.).

\section{Results}

The lithology, stratigraphy, grain size, facies (units), shell frequency and age estimates are summarised in Fig. 2. Analytical results are given in Tables 1-4 and Appendix 1. In the borehole five lithological units were recognised $(A-E)$. The units were assigned to the Maassluis Formation, Koewacht Formation, Kreftenheye Formation, Boxtel Formation and Naaldwijk/ Nieuwkoop Formations (Fig. 2).

\section{Unit A (36.5-62 m; Maassluis Formation)}

The unit consists of fine to middle-grained grey sands with silt in some intervals (Table 1). Light brown and brown-grey clay 


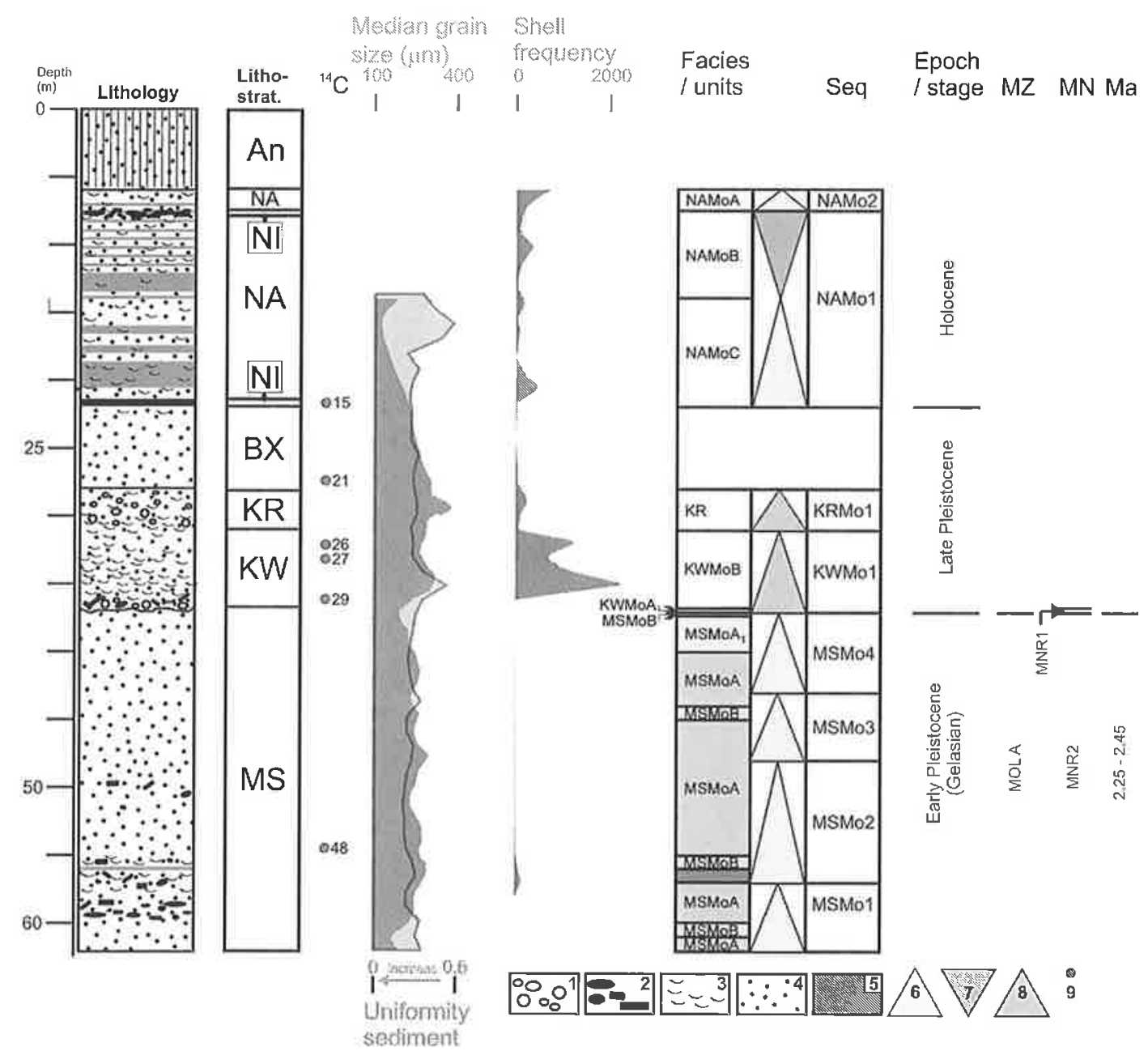

Figure 2 Lithostratigraphic framework of the Moriaanshoofd borehole. 1 - Gravel/pebbles; 2 - Peat and wood pieces; 3 - Shells and shell fragments; 4 - Sand; 5 - Clay; 6 - Deepening trend / transgression; 7 - Shallowing trend / regression / progradation; 8 - Fluvial sequences; 9 - Samples ${ }^{14}$ C-dated; Uniformity sediment - coefficient judging sorting; Seq - Sequence; Depth $(\mathrm{m})$ - Metres below surface; Lithostrat. - Lithostratigraphy; ${ }^{14} \mathrm{C}-$ Radiocarbon dating, numbers correspond to sample numbers in Table 2; Seq - Depositional sequences; MZ - Mollusc zone cf. Spaink (1975); MN - Mammal zone cf. Tesakov (2004); Ma - Age (million years) as indicated by mammal assemblages; An - Anthropogenic; NA - Naaldwijk Formation; NAMOA, NAMOB, NAMOC - Facies in the Naaldwijk Formation; NAMO1, NAMO2 - Sequences in the Naaldwijk Formation; NI - Nieuwkoop Formation; BX - Boxtel Formation; KR - Kreftenheye Formation; KW - Koewacht Formation; KWMOA, KWMOB - Facies in the Koewacht Formation; KWMo1 - Sequence in the Koewacht Formation; MS - Maassluis Formation; MSMoA, MSMOA, MSMOB, MSMoC - Facies in the Maassluis Formation; MSMo1, MSMo2, MSMo3, MSMo4 - Sequences in the Maassluis Formation

fragments and clay pebbles, some of which indurated, and lithic fragments (sandstone, lydite-like) occur in variable amounts. In a small part of the samples shells and shell fragments, often strongly worn, are very common, while most samples are barren (Table 3). Some microvertebrates remains were found (Table 4). Most of the sediment of the unit is free of carbonate. The lower boundary of Unit A was not reached in the borehole.

Several sub-units are recognised, each with distinct features. Each of these sub-units represents a specific depositional environment and is therefore referred to as a facies unit. The sub-units are closely related in terms of sediment properties and textural features, suggesting that they also are closely related in terms of depositional setting.

\section{Interpretation}

The facies properties (Table 1) and their succession are consistent with a shallow marine, tidal-influenced depositional environment (as characterised by Dalrymple, 1994; Reading \& Collinson, 1996 and Van den Berg et al., 2007). Unit A is assigned to the Maassluis Formation of De Mulder et al. (2003).

\section{Facies MSMOA}

The facies consists of rather poorly sorted sand with small amounts of silt. In two intervals the facies is four and three metres thick and in one interval it is ten metres thick (Table 1 and Appendix 1). In interval $48-53 \mathrm{~m}$, some shell fragments, 
Table 1. Facies classification in the record. MSMOA,MSMOA, MSMOB, MSMOC - facies in the Maassluis Formation; KWMOA, KWMOB - facies in the Koewacht Formation; NAMOA, NAMOB, NAMOC - facies in the Naaldwijk Formation.

\begin{tabular}{|c|c|c|c|c|}
\hline $\begin{array}{l}\text { Facies/ } \\
\text { units }\end{array}$ & $\begin{array}{l}\text { Depth } \\
\text { (m b.s.) }\end{array}$ & Lithology & $\begin{array}{l}\text { Median } \\
\text { grain size } \\
(\mu \mathrm{m})\end{array}$ & $\begin{array}{l}\text { Interpreted } \\
\text { environment }\end{array}$ \\
\hline NAMOA & $6-8$ & $\begin{array}{l}\text { Sand, grey, numerous thin clay layers in the upper part; clay, grey with laminated } \\
\text { sandy partings in the lower part; some shells }\end{array}$ & & Supratidal \\
\hline NI & $7.75-8$ & Peat, black, with wood remains & & Peat swamp \\
\hline NAMoB & $8-14$ & $\begin{array}{l}\text { Clay, grey, in some parts laminated (intercalated) with sand, slightly silty; shell } \\
\text { fragments }\end{array}$ & & $\begin{array}{l}\text { High intertidal to } \\
\text { supratidal mud flat (or } \\
\text { possibly salt marsh) }\end{array}$ \\
\hline NAMOC & $14-22$ & $\begin{array}{l}\text { Sand, grey, medium fine- to medium coarse-grained that grades into partially laminated } \\
\text { sandy clays; some shell fragments and shell debris }\end{array}$ & $125-214$ & $\begin{array}{l}\text { Subtidal to } \\
\text { intertidal sand flat }\end{array}$ \\
\hline NI & $21.7-22$ & Peat; dark brown & & Peat swamp \\
\hline KWMOB & $31-36.5$ & $\begin{array}{l}\text { Sand, grey, medium coarse to coarse-grained; some clay clasts; very abundant shells; } \\
\text { some peat fragments and wood pieces }\end{array}$ & $288-321$ & Fluvial deposition \\
\hline KWMoA & 36.5 & $\begin{array}{l}\text { Layer of sand, light grey-beige, fine to medium coarse-grained, poorly sorted, strongly } \\
\text { silty; rounded, brown claystone clasts with a diameter of up to few centimetres and some } \\
\text { very fine gravel consisting of black, lithic fragments; some teeth of small vertebrates }\end{array}$ & 220 & Fluvial deposition \\
\hline MSMOA & $\begin{array}{l}40-44 \\
45-55 \\
57-60 \\
61-62\end{array}$ & $\begin{array}{l}\text { Sand, grey to dark grey, fine to medium coarse, grains subangular to rounded, rather } \\
\text { poorly sorted to well sorted in some parts, silty; clay clasts mainly irregular in shape } \\
\text { in various amounts; some glauconite; some black lithic clasts in some parts; non } \\
\text { calcareous; may contain some: shell fragments, (worn) foraminifers, peat fragments, } \\
\text { wood pieces, some scarce remains of sea-urchins and fishes }\end{array}$ & $189-299$ & Tidal channel fill \\
\hline $\mathrm{MSMOA}_{1}$ & $37-40$ & $\begin{array}{l}\text { Sand, rather dark grey, rather fine to medium coarse, poorly sorted to rather well sorted, } \\
\text { large amounts of fine mica flakes; some fine sandy clay laminae, some light grey } \\
\text { consolidated clay clasts; some peat fragments, some foraminifers and few bone fragments }\end{array}$ & $191-228$ & Tidal channel fill \\
\hline MSMoB & $\begin{array}{l}36.5-37 \\
44-45 \\
55-56 \\
60-61\end{array}$ & $\begin{array}{l}\text { Sand, light grey with brownish beige shade, medium fine- to medium coarse-grained, } \\
\text { rather poorly sorted, strongly silty; may contain some small shell fragments and some } \\
\text { land derived plant material as peat fragments and wood pieces }\end{array}$ & $169-293$ & $\begin{array}{l}\text { Tidal deposits; } \\
\text { possibly tidal channel } \\
\text { upper flanks (edges) } \\
\text { - setting outside tidal } \\
\text { channel bedding }\end{array}$ \\
\hline MSMoC & $56-57$ & $\begin{array}{l}\text { Sand, light beige-grey, medium coarse-grained, grains rounded to subangular, rather } \\
\text { poorly sorted, slightly silty; some very fine quartz gravel, some glauconite, some clay } \\
\text { clasts; large amounts of shell fragments angular in shape }\end{array}$ & 279 & $\begin{array}{l}\text { Lag of tidal gully } \\
\text { (main tidal channel } \\
\text { within the tidal flat } \\
\text { channels network) }\end{array}$ \\
\hline
\end{tabular}

foraminifers and echinoderm spines occur. Interval 40-44 m contains some bone fragments and small amounts of shell fragments.

\section{Interpretation}

This facies is interpreted as tidal channel fills. The lowermost interval of this facies is 61 to $62 \mathrm{~m}$, at the base of the borehole so that the complete thickness of that interval is uncertain. The poorly sorted sand with some silt could reflect deposition on a sand flat or outer parts of a mixed flat (cf. Fig. 12, p. 201 in Dalrymple, 1994; Reading \& Collinson, 2006; Van den Berg et al., 2007).

\section{Facies $\mathrm{MSMOA}_{1}$}

The facies occurs in the upper part of the Maassluis succession (37-40 m). The facies is similar to facies MSMoA in terms of composition and origin (Table 1). An important difference is that facies $\mathrm{MSMOA}_{1}$ consists of generally finer grained (Table 1) poorly to rather well sorted sand, and contains large amounts of very fine mica grains.

\section{Interpretation}

The facies is interpreted as a tidal channel fill. 


\section{Facies MSMOB}

The facies is up to one metre thick and consists of poorly sorted silty sand containing clay clasts angular in shape with sharp edges and also rounded clay clasts. Clay clasts have similar dimensions and are mostly coated by sand grains.

\section{Interpretation}

The grain-size composition and the occurrence at the top of the presumed channel fills are consistent with a derivation from laminated tidal sediments or flaser bedding (or tidal-fluvial zone as defined in Van den Berg et al., 2007). Medium coarse to coarse sands in the interval $55-56 \mathrm{~m}$ could point to tidal channel deposition. Sedimentary structures are not seen in the samples so that more detailed interpretations of the depositional setting are not possible.

Some of the clayey clasts may represent reactivated mud drapes but possibly much of the mud had been transported in the form of faecal pellets. The irregular, sharp edged clasts are presumed to result of fragmentation of thin mud layers during the drilling process.

\section{Facies MSMOC}

This facies occurs only at 56-57 $\mathrm{m}$ depth and consists of a shell bed. The sediment consists of coarse particles, clay clasts and numerous fragments of shells (angular in shape). The shell fragments are strongly abraded and represent a low-diversity suite of coastal species (such as Littorina saxatilis, Nucella lapillus, Mytilus trossulus) (Table 3).

\section{Interpretation}

The facies is interpreted as a basal scour lag of a tidal channel.

\section{Depositional sequences}

The Maassluis Formation interval in Moriaanshoofd Borehole is composed of four depositional sequences (MSMo1-MSMo4, Fig. 2) made up of facies successions/associations that represent lateral migration of depositional tidal systems. Lateral migration of tidal channels leads to the formation of fining-upward sequences (see in Dalrymple, 1994; Reading \& Collinson, 1996 and Johnson \& Baldwin, 1996).

Sequence MSM01 (62-57 m) covers deposits of tidal channel fill / tidal flat. Interval $57-56 \mathrm{~m}$ is a lag deposit and therefore, per definition, a major boundary or unconformity. This interval is interpreted to form a basis of a sequence MSMo2 wherein the lag deposit is overlain by the deposits of tidal channel upper part.

Sequence MSMO3 (43-49 m) covers tidal channel basal part to tidal channel fill deposits. Sequence MSMo4 (36.5-43 m) comprises deposits of tidal channel fill to tidal flat. The four sequences reflect transgressive trends within the depositional setting.

\section{Age}

The mollusc fauna in the interval 55-62 m (Table 3) is consistent with Mol A (Hydrobia ulvae-Mya arenaria) zone of Spaink (1975) indicative of a Gelasian age (Fig. 2).

Arvicolid remains with several Mimomys species from the Maassluis Formation (Table 4) represent a Gelasian assemblage. Notably Mimomys praepliocaenicus and $M$. tigliensis indicate a Gelasian age (see also discussion) and allow the assemblage to be placed in the biozone MNR2 of the system of Tesakov (2004), indicating an age of ca 2.25-2.45 Ma (Fig. 2, see discussion below). We consider the ${ }^{14} \mathrm{C}$ age of $>47,200 \mathrm{BP}$ recorded at $54-55 \mathrm{~m}$ to be at or below the detection limit of radiocarbon dating.

\section{Unit B (31-36.5 m; Koewacht Formation)}

The deposits in the interval are composed of light grey, coarsegrained sands containing some gravel and abundant indurated clay pebbles in its basal part. Shells, mostly well-preserved, are common to very abundant. The deposits abruptly overlay the finer-grained sands of Unit A that lacks shells in its upper parts. Two lithological units are recognised (Table 1) forming one depositional sequence (KWMo1) (Fig. 2).

At the base, at $36.5-36 \mathrm{~m}$ a silty sand layer occurs containing rounded, brown claystone clasts with a diameter of up to few centimetres and some very fine gravel consisting of black, lithic fragments. The sand is fine to medium coarse-grained and poorly sorted (unit KWMOA). The basal interval contains an assemblage of only rooted arvicolids, including Mimomys tigliensis and $M$. reidi (biozone MNR1 of Tesakov, 2004: ca 2.1-2.25 Ma). The basal lag is overlain by a very shell-rich interval (31-36.5 m) (unit $\mathrm{KWMoB}$ ). Shallow marine molluscs, especially shells of Macoma balthica, are abundant and mostly well-preserved. However, differential preservation, and taxa with incompatible ecological (Table 3) as well as stratigraphic signatures occur. A small fraction of the shell fauna consists of Middle Pleistocene, Gelasian, Pliocene and Eocene taxa. The latter includes Homalaxis cf. serratus, Haustator solanderi, Limopsis granulata, Claibornicardia aalterensis and Arcturellina sp.

\section{Interpretation}

Unit B is assigned to the Koewacht Formation of Kiden (2010). The rounded nature of the claystones and the occurrence of ecologically and stratigraphically incompatible taxa combined with the very well-rounded sand grains that are incorporated from earlier reworked and sorted sediments, all point to reworking and deposition by currents. The Eocene taxa, more abundant than the Pliocene taxa, indicate a fluvial (proto-Schelde) 
provenance from the Ghent region (Belgium) where Eocene taxa are found at and near the surface (see discussion). The predominance of well-preserved coastal marine mollusc taxa is attributed to reworking in proximal setting of probably Eemian (MIS 5e) marine deposits.

\section{Age}

The ${ }^{14} \mathrm{C}$ ages (33,220-49,820 BP) (Table 2) of the Koewacht Formation show no straightforward age-depth relationship and one sample is very close to the lower limit of the ${ }^{14} \mathrm{C}$ dating range (Briant et al., 2005). The formation must postdate the youngest ${ }^{14} \mathrm{C}$ age found, viz. $33 \mathrm{ka}$ (see discussion below).

Between 33-35 m two teeth of the genus Microtus were found (Table 4). Their morphology indicates a Late Pleistocene or Holocene age. In the interval from 35 to $36.5 \mathrm{~m}$ an assemblage of only rooted arvicolids occurs, including Mimomys tigliensis and $M$. reidi. It differs from the underlying Maassluis Formation Gelasian assemblage by the presence of teeth definitely attributable to $M$. pliocaenicus and the absence of teeth definitely attributable to $M$. praepliocaenicus. This basal lag assemblage is referred to biozone MNR1 of Tesakov (2004: ca 2.1-2.25 Ma) (Fig. 2). Some arvicolid specimens have a relatively fresh unrolled (i.e. not-damaged) and little mineralised appearance with preservation of fine surface details. This suggests an origin from Early Pleistocene freshwater sediments, indicating reworking and redeposition. No characteristic Middle or Late Pleistocene vole species were recorded in the basal part of the interval. The vast majority of the mollusc fauna indicates a Late Pleistocene age. Strongly abraded EarlyMiddle Pleistocene taxa, such as Corbicula, also occur.

The Middle and Late Pleistocene fluvial sediments in the southwest Netherlands that are deposited by the Schelde River system are assigned to the Koewacht Formation (Kiden, 2010) (see further in discussion).

In works predating Kiden (2010) deposits of the Schelde River have been assigned to a variety of lithostratigraphic units and ages. The Koewacht Formation partly replaces the following lithostratigraphic units (in parentheses literature references in which previously defined units were referred to): Vlissingen deposits (Van Voorthuysen, 1957), Schouwen Formation (Van

Table 2. Radiocarbon ${ }^{14} \mathrm{C}$ ages.

\begin{tabular}{lllll}
\hline $\begin{array}{l}\text { Sample } \\
\text { number in } \\
\text { the borehole surface) }\end{array}$ & $\begin{array}{l}\text { Depth } \\
\text { (m below }\end{array}$ & ${ }^{14} \mathrm{C}$ age (BP) & $\begin{array}{l}\text { Calibrated Sample } \\
\text { age (BP) }\end{array}$ & $\begin{array}{l}\text { number at the } \\
(95 \%)\end{array}$ \\
\hline 15 & $21-22$ & $7680 \pm 40$ & $8550-8400$ & KIA-40438 \\
21 & $27-28$ & $37,950+970-870$ & KIA-40439 \\
26 & $32-33$ & $33,220+540-510$ & KIA-40440 \\
27 & $33-34$ & $49,820+5390-3200$ & KIA-40441 \\
29 & $35-36$ & $35,240+690-640$ & KIA-40443 \\
48 & $54-55$ & $>47,200$ & KIA-40445 \\
\hline
\end{tabular}

Rummelen, 1965), Schouwen deposits within Eem Formation and Eem Formation (Van Rummelen, 1970, 1978), Twente Formation and Kreftenheye Formation (Doppert et al., 1975) and Boxtel Formation (Schokker, 2003).

The Koewacht Formation, as defined by Kiden (2010), was deposited from Saalian (Middle Pleistocene) to Weichselian (Late Pleistocene) times.

\section{Unit C (28-31 m; Kreftenheye Formation)}

The deposits in this unit consist of grey sands. In the lower part sand is coarse-grained and contains common shells. At the bottom some fine gravel and clay clasts occur. In the upper part sand is medium to coarse-grained. Few fine shell fragments occur. Grains of pink quartz within the deposits are very common and the sand grains as well as the claystones are well-rounded.

\section{Interpretation}

The features of sediments point to the Kreftenheye Formation (Busschers \& Weerts, 2003; Busschers et al., 2007; Hijma et al., 2012). The Kreftenheye Formation as defined in Busschers and Weerts (2003) is considered to be deposited by (precursors) of the Rhine between Late Saalian and Early Holocene, but mostly during the Weichselian. Here, the deposits are assigned to one depositional sequence KRMo1 (Fig. 2).

The Unit C (Kreftenheye Formation) in the record at Moriaanshoofd overlays Unit B (Koewacht Formation) and is therefore of younger age ( $<33 \mathrm{ka})$.

\section{Unit D (22-28 m; Boxtel Formation)}

The Boxtel Formation in the Moriaanshoofd Borehole is composed of fine-grained to coarse-grained well-rounded sands. Shells are lacking apart from an odd fragment that probably represents contamination from overlying intervals. Fragments of wood and peat occur.

The Boxtel Formation abruptly overlies the coarser shelly sands of the Kreftenheye Formation. A fining-up trend is found in the sediments.

\section{Interpretation}

The Boxtel Formation represents local small-scale fluvial and/ or fluvio-aeolian deposition in a cold climate setting (Schokker, 2003; Schokker et al., 2005, 2007).

\section{Age of the Boxtel Formation}

Sample $27-28 \mathrm{~m}$ b.s. yielded a ${ }^{14} \mathrm{C}$ age of $37,950 \mathrm{BP}$ (Table 2). Its stratigraphic position in the borehole indicates a much younger age (see discussion). 
Table 3. Mollusc occurrences.

\begin{tabular}{|c|c|c|c|c|c|c|c|c|c|c|c|c|c|c|c|c|c|c|c|c|c|c|c|c|c|c|c|c|c|c|c|c|c|c|c|}
\hline Depth (m) & $\stackrel{\infty}{\perp}$ & $\begin{array}{l}\dot{1} \\
\infty\end{array}$ & 옴 & 공 & $\underset{\exists}{\exists}$ & $\underset{\sim}{\stackrel{m}{~}}$ & $\underset{⿱ 亠}{\dot{m}}$ & $\stackrel{4}{+}$ & 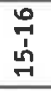 & $\overrightarrow{\vec{b}}$ & $\underset{7}{\stackrel{\infty}{a}}$ & $\begin{array}{l}\text { ஸे } \\
\text { ڤ̆ }\end{array}$ & ì & $\underset{\mathfrak{N}}{\stackrel{N}{N}}$ & $\begin{array}{l}\text { ָे } \\
\text { N } \\
\end{array}$ & $\begin{array}{l}\stackrel{N}{N} \\
\stackrel{N}{N}\end{array}$ & Nิ & 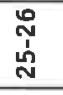 & 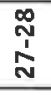 & $\begin{array}{l}\text { స్ } \\
\text { జ } \\
\text { N }\end{array}$ & $\begin{array}{l}\text { ก } \\
\text { ஸे }\end{array}$ & $\vec{m}$ & 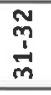 & $\underset{m}{m}$ & $\begin{array}{c}m \\
m \\
m \\
m\end{array}$ & 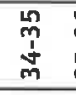 & 望 & 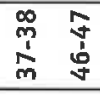 & 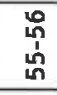 & 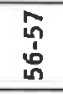 & 隽 & 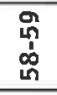 & $\begin{array}{l}8 \\
\vdots \\
0 \\
1\end{array}$ & $\begin{array}{l}-1 \\
\vdots \\
0\end{array}$ & $\begin{array}{l}\tilde{W} \\
i \\
0\end{array}$ \\
\hline Polyplacophora indet. & & & & & & & & & & & & & & & & & & & & & & & & & & 1 & 2 & & & & & & & & \\
\hline Gibbula indet. & & & & & & & & & & & & & & & & & & & & & 1 & 1 & & 1 & 1 & & & & & & & & & & \\
\hline Theodoxus fluviatilis & & & & & & & & & & & & & & 1 & & & & & & & & & & & & & & & & & & & & & \\
\hline Viviparus indet. & & & & & & & & & & & & & & & & & & & & & & & & & & 1 & & & & & & & & & \\
\hline Valvata piscinalis & & & & & & & & & & & & & & 1 & & & & & & & & & & 1 & & 1 & 4 & & & & & & & & \\
\hline Bythina tentaculata opercula & & & & & & & & & & & & & & 13 & & & & & & & & 1 & & 1 & 9 & 13 & 48 & & & & & & & & \\
\hline Bythina tentaculata & & & & & & & & & & & & & & 4 & & & & & & & & & & & & & & & & & & & & & \\
\hline Turritella communis & & & & & & & & & & & & & & & & & & & & & 1 & & & & 1 & & & & & & & & & & \\
\hline Haustator solanderi & & & & & & & & & & & & & & & & & & & & & & 1 & & & 5 & 1 & 5 & & & & & & & & \\
\hline Littorina saxatilis tenebrosa & 131 & 3 & & & & & & & & & & & & 3 & 1 & & & & & & & & & & & & & & & & & & & & \\
\hline Littorina saxatilis/littorea & & & & & & & & & & & & & 1 & & & & & & & 1 & 6 & & & 4 & 4 & 8 & 7 & & 6 & 26 & 3 & & & & \\
\hline Lacuna vincta & & & & & & & & & & & & & & & & & & & & & & & & 9 & 1 & & 11 & & & & & & & & \\
\hline Peringia ulvae & 5 & 23 & 61 & 47 & 222 & 86 & 18 & 6 & 2 & 16 & 4 & 2 & 86 & 248 & 4 & & 1 & 1 & & 4 & 28 & 27 & 2 & 200 & 81 & 1503 & 300 & & & & & & & & \\
\hline Ecrobia ventrosa & 500 & 200 & & & & & & & & & & & 4 & 35 & 1 & & & & & & & 1 & & 51 & 13 & $44 \varepsilon$ & 88 & & & & & & & & \\
\hline Rissoa parva interrupta & & & & & & & & & & & & & & & & & & & & & & & & 20 & 15 & 5 & 5 & & & & & & & & \\
\hline Tornus subcarinatus & & & & & & & & & & & & & & & & & & & & & & & & 1 & & & 3 & & & & & & & & \\
\hline Homalaxis cf. serratus & & & & & & & & & & & & & & & & & & & & & & & & & & & 1 & & & & & & & & \\
\hline Caecum indet. & & & & & & & & & & & & & & 1 & & & & & & & & & & & & & & & & & & & & & \\
\hline Epitonium clathrus & & & & & & & & & & & & & & & & & & & & & 1 & & & & 1 & & 1 & & & & & & & & \\
\hline Euspira hemiclausa & & & & & & & & & & & & & & & & & & & & & & & & & 1 & & 4 & & & & & & & & \\
\hline Nucella lapillus & & & & & & & & & & & & & & & & & & & & & & & & & & & & & & 6 & & & & & \\
\hline Buccinum undatum & & & & & & & & & & & & & & & & & & & & & & & 1 & 1 & & 1 & 1 & & & & & & & & \\
\hline Hinia reticulatus/nitidum & & & & & & & & & & & & & & & & & & & & & & & & 1 & & 1 & 3 & & & & & & & & \\
\hline Hinia pygmaea & & & & & & & & & & & & & & & & & & & & & & & & & 1 & & 2 & & & & & & & & \\
\hline Amyclina labiosa & & & & & & & & & & & & & & & & & & & & & & & & & & & 1 & & & & & & & & \\
\hline Oenopota turricula & & & & & & & & & & & & & & & & & & & & & 1 & & & 1 & 1 & & 2 & & & & & & & & \\
\hline Turbonilla crenata & & & & & & & & & & & & & & & & & & & & & 1 & & & & & & & & & & & & & & \\
\hline Turbonilla sp.1 & & & & & & & & & & & & & & & & & & & & & & & & 2 & & 2 & & & & & & & & & \\
\hline Turbanilla sp.2 & & & & & & & & & & & & & & & & & & & & & & & & 1 & & & & & & & & & & & \\
\hline Retusa obtusa & & & & & 2 & & 1 & & & & & & 3 & 9 & & & & & & & & 1 & & 2 & 1 & & 14 & & & & & & & & \\
\hline Haminoea navicula & & & & & & & & & & & & & & & & & & & & & & 1 & & & & & & & & & & & & & \\
\hline Anisus leucostoma & & & & & & & & & & & & & & & & & & & & & & & & & & & 1 & & & & & & & & \\
\hline Planorbis planorbis & & & & & & & & & & & & & & & & & & & & & & & & 1 & & & & & & & & & & & \\
\hline Gyraulus crista & & & & & & & & & & & & & & & & & & & & & & & & 1 & & & & & & & & & & & \\
\hline
\end{tabular}


Table 3 continued.

\begin{tabular}{|c|c|c|c|c|c|c|c|c|c|c|c|c|c|c|c|c|c|c|c|c|c|c|c|c|c|c|c|c|c|c|c|c|}
\hline Depth $(\mathrm{m})$ & $\stackrel{\infty}{1}$ & $\begin{array}{l}0 \\
0 \\
0\end{array}$ & ํํㅁ & $\underset{ }{\stackrel{1}{\prime}}$ & $\begin{array}{l}N \\
\stackrel{7}{-} \\
\stackrel{-}{-}\end{array}$ & $\underset{\sim}{\stackrel{m}{a}}$ & $\stackrel{ \pm}{m}$ & $\begin{array}{l}n \\
7 \\
\pm\end{array}$ & $\begin{array}{l}0 \\
\stackrel{1}{1} \\
\stackrel{6}{-1}\end{array}$ & $\stackrel{5}{5}$ & $\underset{\sim}{\stackrel{\infty}{1}}$ & 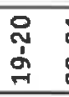 & 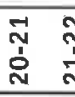 & ָ̃ & 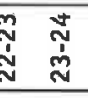 & 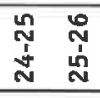 & 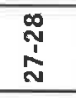 & 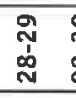 & 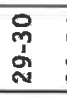 & ⿳ें & $\begin{array}{cc}\mathcal{N} & m \\
& m \\
\dot{m} & m \\
m\end{array}$ & 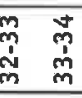 & 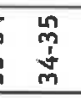 & $\begin{array}{l}\stackrel{\circ}{n} \\
\dot{m} \\
\dot{m}\end{array}$ & 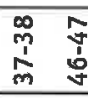 & 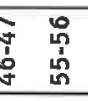 & 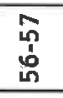 & $\begin{array}{l}\infty \\
\stackrel{1}{\Lambda} \\
\\
\end{array}$ & 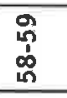 & $\begin{array}{l}8 \\
\vdots \\
1 \\
\text { in } \\
\end{array}$ & $\begin{array}{l}\overrightarrow{0} \\
\vdots \\
0\end{array}$ & $\frac{\mathfrak{1}}{1}$ \\
\hline Gyraulus acronicus & & & & & & & & & & & & & & & & & & & & & & 1 & & & & & & & & & & \\
\hline Gyraulus rossmaesleri & & & & & & & & & & & & & & & & & & & & & & 1 & & & & & & & & & & \\
\hline Galba truncatula & & & & & & & & & & & & & & & & & & & & & & & 2 & & & & & & & & & \\
\hline Stagnicola palustris sl & & & & & & & & & & & & & & & & & & & & & & 1 & & 1 & & & & & & & & \\
\hline Radix peregra/ovata & & & & & & & & & & & & & & & & & & & & & & 1 & & & & & & & & & & \\
\hline Succinea cf. schumacheri & & & & & & & & & & & & & & & & & & & & & & 4 & 1 & 8 & & & & & & & & \\
\hline Succinea oblonga & & & & & & & & & & & & & & & & & & & & 1 & & 6 & & & & & & & & & & \\
\hline Succinea elegans/putris & & & & & & & & & & & & & & & & & & & & & & 1 & & & & & & & & & & \\
\hline Columella columella & & & & & & & & & & & & & & & & & & & & & & 2 & & 1 & & & & & & & & \\
\hline Vertigo genesii & & & & & & & & & & & & & & & & & & & & & & 2 & & & & & & & & & & \\
\hline Pupilla muscorum & 2 & & & & & & & & & & & & & & & & & & & & & 1 & 1 & 2 & & & & & & & & \\
\hline Vallonia pulchella & 8 & & & & & & & & & & & & & & & & & & & & & 1 & & & & & & & & & & \\
\hline Trochulus hispidus & & & & & & & & & & & & & & & & & & & & & & 1 & & 1 & & & & 1 & & & & \\
\hline Candidula indet. & 1 & & & & & & & & & & & & & & & & & & & & & & & & & & & & & & & \\
\hline Helicellinae indet. & 6 & & & & & & & & & & & & & & & & & & & & & & & & & & & & & & & \\
\hline Nucula nucleus & & & & & & & & & & & & & & & & & & & & & & & & 1 & & & & & & & & \\
\hline Nuculoma tenuis & & & & & & & & & & & & & & & & & & & & & & & & 1 & & & & & & & & \\
\hline Acila cobboldiae & & & & & & & & & & & & & & & & & & & & & & & 1 & & & & & & & & & \\
\hline Yoldia indet. & & & & & & & & & & & & & & & & & & & 1 & & & 3 & 3 & 4 & & 1 & 3 & & & & & \\
\hline Limopsis granulata & & & & & & & & & & & & & & & & & & & & & & 1 & & & & & & & & & & \\
\hline Mytilus edulis & 12 & 1 & & 1 & 84 & 28 & 4 & 2 & 4 & 1 & 3 & 1 & 4 & 1 & 1 & 1 & 1 & & 7 & 2 & 2 & $28 \quad 10$ & 17 & 30 & 1 & & & & & & & \\
\hline Mytilus trossulus & & & & & & & & & & & & & & & & & & & & & & & & & & 17 & 70 & 4 & 1 & 1 & 1 & 1 \\
\hline Ostrea edulis & & 1 & & & & & & & & & & & 1 & 2 & & & & & 2 & 1 & 1 & 8 & 11 & 24 & & & & & & & & \\
\hline Cubitostrea indet. & & & & & & & & & & & & & & & & & & & & & & 1 & & & & & & & & & & \\
\hline Heteranomia squamula & & & & & & & & & 5 & & & & & & & & & & & & & & & & & & & & & & & \\
\hline Aequipecten opercularis & & & & & & & & 1 & 4 & 1 & 1 & & & & & & & & & & & & & & & & & & & & & \\
\hline Mimachlamys varia & & & & & & & & & & & & & & & & & & & & & & 2 & 2 & 1 & & & & & & & & \\
\hline Lucinella divaricata & & & & & & & & & & & & & & & & & & & & & & & 1 & 1 & & & & & & & & \\
\hline Diplodonta astartea & & & & & & & & & & & & & & & & & & & & & & & & 1 & & & & & & & & \\
\hline Hemilepton nitidum & & & & & & & & & & & & & & & & & & & & & & 1 & & & & & & & & & & \\
\hline Kurtiella bidentata & 2 & & & 3 & 7 & 5 & & 4 & 14 & 2 & 8 & & 4 & 121 & 1 & & & & & & 50 & 50 & 11 & 48 & & & & & & & & \\
\hline Tellimya ferruginosa & & & & & & & & & & & 2 & & 1 & & & & & & & & & & 1 & & & & & & & & & \\
\hline Arcturellina sp. & & & & & & & & & & & & & & & & & & & & & & 2 & & 3 & & & & & & & & \\
\hline Claibomicardia aalterensis & & & & & & & & & & & & & & & & & & & & & & 1 & 1 & 3 & & & & & & & & \\
\hline Cyclocardia scalaris & & & & & & & & & & & & & & & & & & & & & 1 & 1 & 2 & 5 & & & & & & & & \\
\hline
\end{tabular}




\begin{tabular}{|c|c|c|c|c|c|c|c|c|c|c|c|c|c|c|c|c|c|c|c|c|c|c|c|c|c|c|c|c|c|c|c|c|c|c|}
\hline $\begin{array}{ll}\text { Depth (m) } \\
\end{array}$ & $\stackrel{\infty}{1}$ & $\begin{array}{l}9 \\
1 \\
\infty\end{array}$ & $\begin{array}{l}0 \\
\overrightarrow{1} \\
a\end{array}$ & $\begin{array}{l}-1 \\
7 \\
0 \\
-1\end{array}$ & \begin{tabular}{l}
\multirow{1}{*}{} \\
$\stackrel{-}{-}$ \\
-
\end{tabular} & $\underset{\sim}{\stackrel{m}{二}}$ & $\begin{array}{l}\vec{H} \\
\stackrel{\vec{m}}{\mathrm{~m}} \\
\end{array}$ & 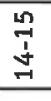 & \begin{tabular}{l}
0 \\
\multirow{1}{*}{} \\
ஸे \\
\end{tabular} & $\begin{array}{c}f \\
\vdots \\
\dot{b} \\
-1\end{array}$ & $\begin{array}{l}\infty \\
\stackrel{1}{1} \\
\end{array}$ & $\begin{array}{l}\stackrel{9}{N} \\
\text { o }\end{array}$ & స̃ & $\underset{\mathfrak{N}}{\stackrel{N}{N}}$ & $\underset{N}{N}$ & Ñ & 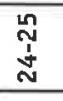 & $\begin{array}{l}\stackrel{0}{N} \\
\stackrel{N}{N}\end{array}$ & 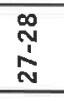 & $\begin{array}{l}\text { N } \\
\text { ஸे } \\
\text { N }\end{array}$ & 嗯 & $\begin{array}{l}\vec{m} \\
\dot{p} \\
\dot{m}\end{array}$ & $\begin{array}{l}\tilde{N} \\
\dot{m} \\
\dot{m}\end{array}$ & $\underset{m}{m}$ & $\begin{array}{l} \pm \\
m \\
m \\
m\end{array}$ & $\begin{array}{l}n \\
m \\
j \\
m \\
m\end{array}$ & $\begin{array}{l}\stackrel{p}{n} \\
n \\
n \\
n\end{array}$ & 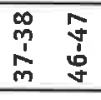 & 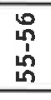 & 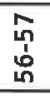 & 菖 & 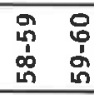 & $\begin{array}{l}\overrightarrow{0} \\
\dot{8} \\
\end{array}$ & $\begin{array}{l}\tilde{o} \\
1 \\
0\end{array}$ \\
\hline Cyclocardia chamaeformis & & & & & & & & & & & & & & & & & & & & & & & & & & & 1 & & & & & & & \\
\hline Goodallia triangularis & & & & & & & & & & & & & & & & & & & & & 1 & & & 2 & & & 4 & & & & & & & \\
\hline Pteromeris corbis & & & & & & & & & & & & & & & & & & & & & & & & & & & 1 & & & & & & & \\
\hline Astarte incerta & & & & & & & & & & & & & & & & & & & & & & & & 1 & & 1 & 3 & & & & & & & \\
\hline Astarte obliquata & & & & & & & & & & & & & & & & & & & & & & & & & & & 1 & & & & & & & \\
\hline Tridonta montagui & & & & & & & & & & & & & & & & & & & & & & & & & & & & & & 1 & & & & \\
\hline Digitaria digitaria & & & & & & & & & & & & & & & & & & & & & & & & & & 1 & 2 & & & & & & & \\
\hline Cerastoderna edule & 8 & 5 & 13 & 8 & 6 & 7 & 3 & 2 & 4 & 3 & 1 & 1 & 21 & 55 & 4 & & & & & 4 & 15 & 7 & 4 & 13 & 33 & 25 & 96 & & & & & & & \\
\hline Cerastoderma glaucum & 15 & & & & & & & & & & & & 1 & 30 & & & & & & & 6 & & & 44 & 2 & 7 & 10 & & & & & & & \\
\hline Serripes groenlandicus & & & & & & & & & & & & & & & & & & & & & & & & & 1 & & 1 & & & & & & & \\
\hline Mactra stultorum & & & & & & & & & & & & & & & & & & & & & 1 & & & 1 & 2 & & 1 & & & & & & & \\
\hline Spisula subtruncata & & & & & & & 7 & 4 & 32 & 2 & 13 & & 1 & 2 & & & & 2 & & 8 & 62 & 13 & 5 & 300 & 130 & 200 & 300 & & & & & & & \\
\hline Spisula elliptica & & & & & & & & & & & & & & & & & & & & & 3 & 2 & 1 & 15 & 49 & 17 & 59 & & & & & & & \\
\hline Solen marginatus & & & & & & & & & & & & & & & & & & & & & & & & & & & 1 & & & & & & & \\
\hline Phaxas pellucidus & & & & & & & & & 2 & & & & & & & & & & & & & & & & & & & & & & & & & \\
\hline Ensis arcuatus & & & & & & & & & & & & & & & & & & & & & & & & & & & 2 & & & & & & & \\
\hline Ensis minor & & & & & & & & & & & & & & & & & & & & & & & & & & & 1 & & & & & & & \\
\hline Ensis indet. & & & & & & & & & & & & & & & & & & & & & & & 1 & 1 & 1 & 1 & 1 & & & & & & & \\
\hline Angulus tenuis & & & & & & & & & 2 & & 4 & & & 1 & & & & & & 1 & 1 & & & 9 & 1 & 12 & 36 & & & & & & & \\
\hline Angulus donacina & & & & & & & & & 4 & & & & & & & & & & & 1 & & & 1 & & & & & & & & & & & \\
\hline Angulus fabula & & & & & & & & & & & & & & & & & & & & 3 & 8 & & & 25 & 18 & 8 & 16 & & 1 & & & & & \\
\hline Angulus pygmaea & & & & & & & & & 1 & & & & & & & & & & & & & & & & & & & & & & & & & \\
\hline Macoma balthica & 5 & & 14 & 14 & 11 & 12 & 1 & 8 & 70 & 5 & 36 & & 21 & 22 & & & & & 1 & 5 & 19 & 6 & 11 & 90 & 105 & 2003 & 300 & & 1 & & & & & \\
\hline Macoma praetenuis & & & & & & & & & & & & & & & & & & & & & 2 & & & 4 & 10 & 5 & 7 & & & & & & & \\
\hline Macoma obliqua & & & & & & & & & & & & & & & & & & & & & & & & 2 & 1 & 6 & 4 & & 1 & & & & & \\
\hline Gastrana fragilis & & & & & & & & & & & & & & & & & & & & & & & & & & 1 & 2 & & & & & & & \\
\hline Donax vittatus & & & & & & & & & & & 1 & & & & & & & & & & 4 & 1 & 2 & 45 & 30 & 641 & 117 & & & & & & & \\
\hline Abra alba & & & & & & & & & 16 & & 3 & & 1 & & & & & & & 6 & 22 & 3 & 3 & 148 & 79 & 902 & 200 & & & & & & & \\
\hline Abra prismatica & & & & & & & & & & & & & & & & & & & & & & & & 2 & 3 & 6 & & & & & & & & \\
\hline Scrobicularia plana & 1 & 3 & 6 & 4 & 11 & 1 & & & & & & & 6 & 10 & & 1 & 1 & & & 3 & 15 & 2 & 4 & 22 & 24 & 21 & 41 & & & & & & & \\
\hline Arctica islandica & & & & & & & & & & & & & & & & & & & & & & & & & 1 & & 1 & & 1 & 8 & 1 & 1 & & \\
\hline Dosinia indet. & & & & & & & & & & & & & & & & & & & & & & & & & & 1 & 1 & & & & & & & \\
\hline Chamelea striatula & & & & & & & & & & & & & & & & & & & & & & & & & & & 1 & & & & & & & \\
\hline Venerupis senescens & & & & & & & & & & & & & & & & & & & 1 & & 2 & 1 & & 3 & 3 & 8 & 5 & & & & & & & \\
\hline Venerupis decussatus & & & & & & & & & & & & & & & & & & & & & & & & & & & 1 & & & & & & & \\
\hline
\end{tabular}


Corbicula fluminalis auct.

2

Pisidium amnicum

Pisidium casertanum

Pisidium henslowanum

Pisidium milium

Pisidium nitidum

Pisidium subtruncatum

Pisidium supinum

Sphaerium corneum

Sphaerium cf. subsolidum

Mya arenaria

Varicorbula gibba

Saxicavella jeffreysi

Pholas dactylus

Barnea candida

Zirfaea crispata

Turitella indet.

Natica indet.

Nassarius indet.

Gastropoda indet.

Nuculidae indet.

Pulmonata sp.

Cyclocardia indet.

Carditidae indet.

Astarte indet.

Spisula indet.

Macoma indet.

Venerupis indet.

Sphaeriidae indet.

Mya indet.

Bivalvia indet.

Succinea indet.

Columella indet

Cerastoderma indet.

Pisidium indet.

$\begin{array}{lllll}1 & 1 & 4 & 1 & 1\end{array}$

$\begin{array}{llll}2 & 4 & 2 & 1\end{array}$

12

$10 \quad 1$

1

18

1

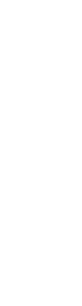

1

1

$\begin{array}{lll}3 & 6 & 2\end{array}$

$1 3 \longdiv { 4 } 5$

$\begin{array}{llll}13 & 4 & 15 & 37\end{array}$

1

2

$\begin{array}{llll}24 & 23 & 28 & 49\end{array}$

$\begin{array}{lll}1 & 1 & 1\end{array}$

$\begin{array}{lll}1 & 7 & 1\end{array}$

111

$\begin{array}{lll}6 & 1 & 13\end{array}$

1

1

2

95100

2

$\begin{array}{lllllll}1 & 1 & 1 & 23 & 3 & 11\end{array}$

11

14

41

$\begin{array}{llllll}2 & 1 & 1 & 8 & 1\end{array}$

$125 \quad 3$

13

1

$37100 \quad 1$ 
Table 4. Mammal occurrences.

\begin{tabular}{lll}
\hline Taxa & Depth (m b.s.) \\
\cline { 2 - 3 } & $33-3636-3737-6254-5555-5656-58$ \\
\hline Mimomys pliocaenicus & $\bullet$ & $\bullet$ \\
Mimomys praepliocaenicus/ & $\bullet$ & $\bullet$ \\
pliocaenicus \\
Mimomys praepliocaenicus \\
Mimomys tigliensis \\
Mimomys reidi \\
Pitymimomys sp. \\
Microtus sp. \\
Microtus agrestis \\
Desmana sp. \\
Soricidae
\end{tabular}

\section{Unit E (<22 m; Naaldwijk/Nieuwkoop Formation)}

The Naaldwijk Formation in the Moriaanshoofd Borehole is composed of grey, fine to coarse-grained sands with an interval of grey clay and finely layered to laminated sand-clay alternations. Shell debris and shells occur in variable amounts. The upper six metres consists of anthropogenic sediments used for dike construction. At the bottom (21.7-22 $\mathrm{m}$ ) and in the interval 7.75-8 $\mathrm{m}$ thin peat layers occur that are attributed to the Nieuwkoop Formation. Three lithological units are recognised (bottom up): NAMOC, NAMoB and NAMOA (Table 1).

\section{Interpretation and age}

The record of the Naaldwijk Formation and the Nieuwkoop Formation combined represent two depositional sequences.

The succession of peat and the overlying facies within the Naaldwijk Formation (NAMoC, NAMoB) form a single depositional sequence (NAMo1) (Fig. 2).

Overlying the basal peat (21.7-22 $\mathrm{m}$ ) is an interval of fine to coarse-grained sands that grade into (partially laminated) sandy clays. The mollusc fauna consists of a mixture of intertidal to shallow subtidal mesohaline taxa (Table 3 ). Above $20 \mathrm{~m}$ b.s. the mollusc fauna (species) becomes fully marine. The interval from 14 to $18 \mathrm{~m}$ is interpreted to cover the high-stand deposits within the Naaldwijk Formation since it contains fully marine mollusc taxa (Table 3) and sandy and sandy/clayey layered sediments that are interpreted to originate in the lower part of the tidal setting. In this view the maximum flooding surface within the formation in the record at Moriaanshoofd occurs at $14 \mathrm{~m}$ depth.

Above $14 \mathrm{~m}$ a shallowing trend is interpreted. The deposits are characterised by silty clay grading into laminated or finely layered fine sand-silt alterations that indicate deposition within the upper tidal flat. Faunas become progressively dominated by shallow water species, and increasingly mesohaline and even oligohaline taxa occur. At the top of the sequence (7.75-8 $\mathrm{m}$ ) a thin peat layer occurs (Nieuwkoop Formation).

The top part of the succession (6-7.75 m; unit NAMoA) contains sandy clays and sands, and is dominated by mesohaline mollusc taxa as well as common remains of terrestrial snails. The unit NAMOA is interpreted to represent a transgressive part of sequence NAMo2. The Naaldwijk Formation forms the Holocene clastic coastal infill in the western Netherlands (Weerts, 2003) and intertongues with peat layers of the Nieuwkoop Formation that formed mainly on the landward side of the coastal plain (Weerts \& Busschers, 2003)

Deposits in the lower part of the Naaldwijk Formation in the Moriaanshoofd record (8-21.7 m) are consistent with the Wormer Member and deposits in the upper part of the Naaldwijk Formation (6-7.75 m) are consistent with the Walcheren Member of the Naaldwijk Formation as defined by Weerts (2003) (see also Hijma et al., 2009 and Westerhoff et al., 2003)

The origin of the Naaldwijk Formation is related to the Holocene transgression that postdated the last glacial period of the Late Pleistocene (see for detailed approach Hijma et al., 2009, 2010; Hijma \& Cohen, 2010 and for the paleogeographic reconstructions Vos et al., 2011).

The Rhine-Meuse paleovalley (Busschers et al., 2007; Hijma et al., 2009 and Hijma \& Cohen, 2011) had been transgressed gradually in the course of Holocene and underwent successive transformation from a river valley to an estuary under the influence of rapidly rising sea-level between $9 \mathrm{ka}$ and $6 \mathrm{ka}$ (Hijma \& Cohen, 2011; Hijma et al., 2009).

According to paleogeographic reconstructions in Hijma \& Cohen (2011) and Hijma et al. (2009) in the area to the southwest of the paleovalley, where Moriaanshoofd is located, tidal basin existed already at $8.5-8.0 \mathrm{ka}$ pointing to transgression in that time period. A single ${ }^{14} \mathrm{C}$ age from the basal peat layer in the Moriaanshoofd record (Table 2) shows that the Holocene transgression reached the Moriaanshoofd area around $7680 \mathrm{BP}$.

\section{Discussion}

\section{: Small mammals refine age estimates for the Maassluis Formation}

The biozonation of the Late Pliocene and Early Pleistocene arvicolid faunas of South Eastern Europe (Russian Federation, Ukraine) defined by Tesakov (2004) is considered applicable to Western Europe. This allows to suggest more precise limits than before for the age of this part of the Maassluis Formation, with the assumption that reworking did not substantially affect the age estimates. A detailed discussion of the taphonomy of these small mammal remains is outside the scope of this paper. The presence of Mimomys praepliocaenicus in Moriaanshoofd indicates biozones MNR2 or MNR3, and the additional occurrence of $M$. tigliensis (at 55-56 m at Moriaanshoofd, sequence MSM02) indicates a date not older than the most recent part of biozone 
MNR2 (age brackets 2.25-2.35 Ma). These age brackets are based on calibration using magnetostratigraphy (Pevzner et al., 1998), which places the arrival of late Allophaiomys faunas between 2-2.1 Ma (biozone MQR11) (see Pevzner et al., 1998).

The small mammals from the Maassluis Formation in the Moriaanshoofd Borehole are similar to those of Zuurland level 11 (Tesakov, 1998; Van Kolfschoten, 1988), assigned to biozone MNR2 (Tesakov, 2004). The Oosterschelde dredged material described by Reumer et al. $(1998,2005)$ contains arvicolids similar to those found here in the lag deposit at 36-36.5 m (biozone MNR1). The arvicolid material from Schelphoek Borehole 40-45 m, Maassluis Formation cycle 6, described by Slupik et al. (2007) consists of only a few teeth, two of which were referred to $M$. pliocaenicus. However these teeth have insufficient features to assign them to that species or to the older M. praepliocaenicus. The Moriaanshoofd material from the Maassluis Formation appears to be somewhat older than the Tegelen arvicolid material with Mimomys pliocaenicus placed in biozone MNR1 (Tesakov, 1998, 2004) (see also Mayhew \& Gibbard, 1998 and Mayhew and Stuart, 1986).

The sequences in the Maassluis Formation interval in the Moriaanshoofd Borehole are not as clearly expressed as in the Schelphoek Borehole. However, the four sequences documented in the present borehole (MSMo1-MSMo4) match quite well the thickness and depth below surface of the upper Maassluis sequences in Schelphoek Borehole (located at about $900 \mathrm{~m}$ distance; Slupik et al., 2007; Fig. 3). The Late Quaternary regional unconformity at the top of the Gelasian deposits is also found at similar depths in both boreholes.

\section{Age of Late Pleistocene successions}

The age estimates of the three Late Pleistocene units (Unit B-D: Koewacht Formation, Kreftenheye Formation and Boxtel Formation) in the borehole are not straightforward. The units must have been deposited after the Eemian interglacial (MIS 5e, $<115 \mathrm{ka}$ ) as typical Eemian mollusc indicator taxa are found in these intervals and prior to the Holocene (MIS 1). The apparent contradictory ${ }^{14} \mathrm{C}$ ages we obtained can be understood as a result of mixing and reworking in these mostly fluvial units and they should provide maximum ages for each of the units. Further correlation with dated units close to the study area constrains the age estimates of each of the units further.

The youngest ${ }^{14} \mathrm{C}$ age obtained for a peat sample from the Koewacht Formation in Moriaanshoofd indicates that this unit must have been deposited after 33 ka (late Middle Pleniglacial or younger). De Koewacht Formation in borehole Moriaanshoofd can be correlated to Unit B4 in the offshore region to the northwest of study site (Hijma et al., 2012 following Busschers et al., 2007) whose age is estimated between 48-30 ka (late Middle Pleniglacial). The B4 Unit of Hijma et al. (2012) represents fluvial deposits of the Rhine-Meuse River system and is assigned to the Kreftenheye Formation, the Koewacht Formation

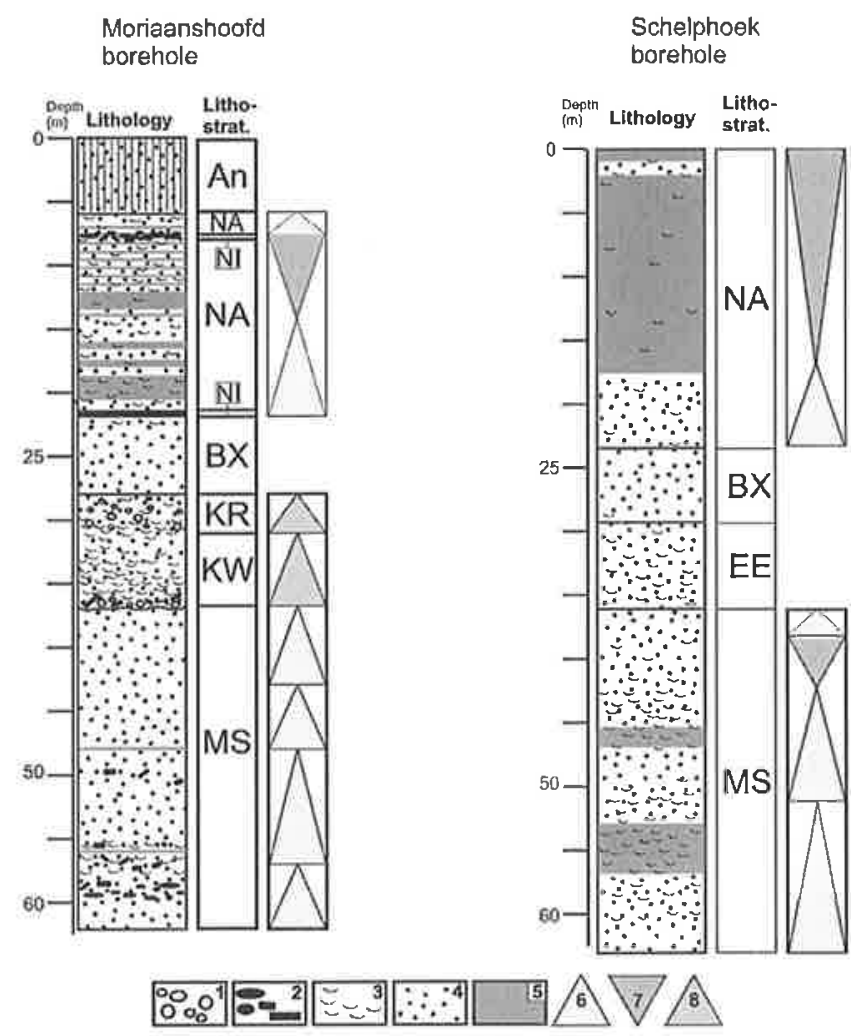

Fig. 3. Correlation of the Quaternary successions in Moriaanshoofd and Schelphoek boreholes. 1 - Gravel/pebbles; 2 - Peat and wood pieces; 3 - Shells and shell fragments; 4 - Sand; 5 - Clay; 6 - Deepening trend/ transgression; 7 - Shallowing trend / regression / progradation; 8 - Fluvial sequences; Depth (m) - Metres below surface; Lithostrat. - Lithostratigraphy; An - Anthropogenic; NA - Naaldwijk Formation; NI - Nieuwkoop Formation; EE - Eem Formation; $B X$ - Boxtel Formation; KR - Kreftenheye Formation; KW - Koewacht Formation; MS - Maassluis Formation.

represents coeval paleo-Schelde deposition. The three inconsistent ${ }^{14} \mathrm{C}$ ages obtained from the Koewacht Formation confirm the mixed (reworked) origin for this unit already shown by the fossil content.

The age of the Kreftenheye Formation interval in Moriaanshoofd is older than the maximum age ( $33 \mathrm{ka}$ ) of the underlying Koewacht Formation. Correlation of the Kreftenheye Formation interval from Moriaanshoofd with deposits offshore (Hijma et al., 2012) or in the Rhine-Meuse paleovalley onshore (Busschers et al., 2007) is difficult. The Kreftenheye interval in Moriaanshoofd might correspond to onshore units B5 and B6 of Busschers et al. (2007) that are lumped into a single offshore unit (Hijma et al., 2012). But correlation with the uppermost part of Unit B4 of Hijma et al. (2012) can not be excluded. The depth of the Moriaanshoofd Kreftenheye interval (28-31 m = 23.81-26.81 m NAP) fits best a correlation with the uppermost part of unit B4 or lower part of unit B5 of Hijma et al. (2012). These units correspond to a major southward shift of the Rhine-Meuse system that occurred during the later phase of Middle Pleniglacial to early Late Pleniglacial (around the MIS 3/2 boundary: 30-25 ka, Busschers et al., 2007). 
The Boxtel Formation (Unit D; 22-28 m) in Moriaanshoofd is very similar as the interval in nearby borehole Schelphoek (Slupik et al., 2007). In the Rhine-Meuse paleovalley to the northeast of Moriaanshoofd, the formation is represented by river-dunes (Busschers et al., 2007 and Hijma et al., 2009; their unit B6c).

These aeolian deposits yielded age estimates between ca 11 and $15 \mathrm{ka}$, placing them after the Last Glacial Maximum (LGM) in MIS 2 (Busschers, 2008). However, we cannot exclude that the fluvial reworked aeolian deposits of the Boxtel Formation at Moriaanshoofd are older than the deposits in the RhineMeuse paleovalley.

\section{A proto-Schelde River deposit disguised as a marine interval}

The shelly interval overlying the truncation of the Gelasian in the Moriaanshoofd Borehole (28-36.5 m) (base of the Koewacht Formation) consists mostly of reworked marine sediments, predominantly derived from local Eemian deposits, is interpreted as a fluvial deposit. The rare Eocene mollusc species derive from the Late Ypresian-Early Lutetian Aalter Formation that crops out in a zone located from Ghent to Brugge to the North Sea off Zeebrugge, Belgium (Steurbaut \& Nolf, 1989; Du Four et al., 2006) (Fig. 4). These Eocene taxa therefore indicate an ancient Schelde River that drained northwards from the area around Ghent in Belgium. The modern Schelde River via Antwerp, that became established during the latest Weichselian (Tavernier \& De Moor, 1974; Kiden, 1991), eroded and reworked substantial amounts of Miocene and Pliocene sediments and fossils in the vicinity of Antwerp (Kiden, 2006). These modern

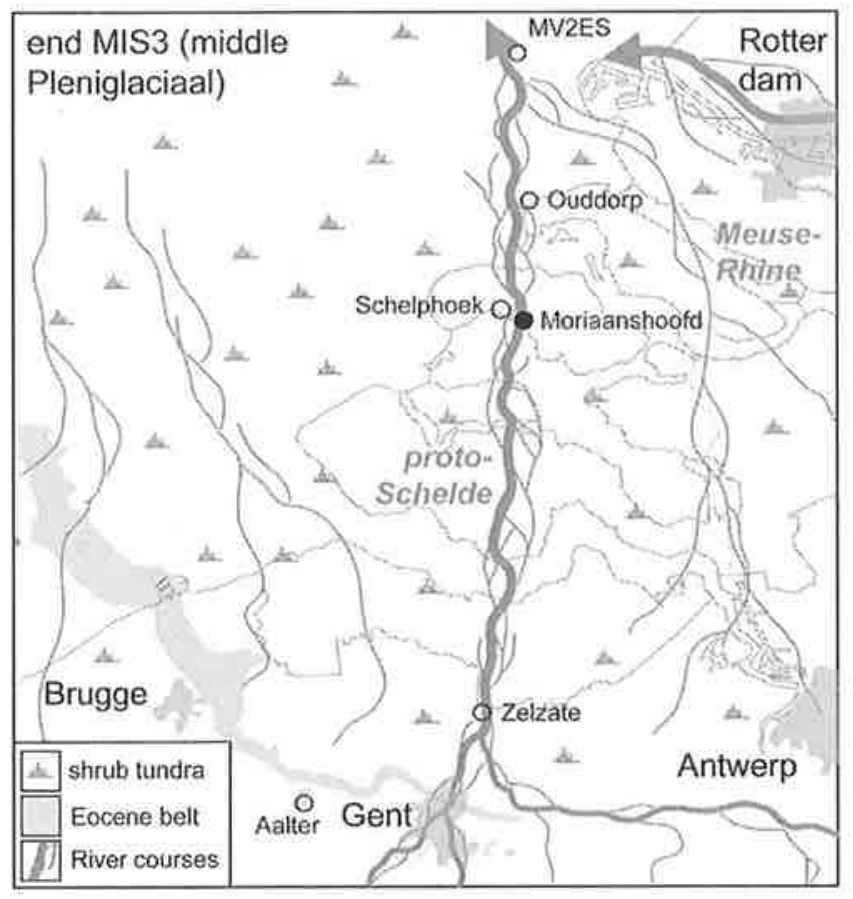

Fig. 4. Paleogeography of the Flemish Schelde River.
'Schelde Sands' are characterised by an abundance of reworked Neogene fossils that are very rare in the area studied here. Low numbers of Pliocene taxa found in the Moriaanshoofd Borehole (e.g. Astarte incerta, A. obliquata, Cyclocardia scalaris, Amyclina labiosa) probably derive from the current Westerschelde area. There, Pliocene deposits located at shallow depth have been eroded by Pleistocene rivers. Fluvial deposits containing reworked Eocene taxa probably derived from the Aalter Formation are known from the 'Vlaamse Vallei' near Zelzate (Janssen, 1965). Low numbers of Eocene taxa in the absence of many Neogene taxa are also known from dredging activities in the Hammen and the North Sea off Rotterdam, as well as from the beaches near Ouddorp (De Bruyne et al., 1987). The most northerly reference point for fluvial deposits containing Eocene taxa, probably derived from the Aalter Formation, is a borehole near The Hague (Meijer and Cleveringa, 2009).

A shelly interval similar to that found in the Kreftenheye Formation in the Moriaanshoofd Borehole was found in the Schelphoek Borehole (29-34 m). The latter was attributed to either an Eemian or Middle Pleistocene marine setting (Slupik et al., 2007). There, very low amounts of freshwater taxa as well as oligohaline (0.5-5 per mil) taxa occur in an otherwise polyhaline (18-30 per mil) fauna, also suggest reworking. No stratigraphically older species were found in the Schelphoek Borehole, but nevertheless the shelly interval there presumably should be attributable to the Kreftenheye Formation.

Reworking of deposits and faunas is extensive in Quaternary intervals in SW Netherlands. North of the study area, in the vicinity of Rotterdam, Busschers et al., (2007) found evidence of increased erosive fluvial activity of the Maas-Rhine River system during the late middle to early Late Pleniglacial (MIS 3: ca $24-30 \mathrm{ka}$ ). Increased erosive fluvial activity occurred repeatedly during the Weichselian. At the transition MIS 5a-4 (ca $75 \mathrm{ka}$ ) sea level dropped considerably from $\sim 25$ to $90 \mathrm{~m}$ below present levels, triggering fluvial reworking (Hijma et al., 2012). MIS 4 (75-60 ka) was a period of extensive fluvial incisions across NW Europe including the Rhine and Meuse and also the Flemish Valley. The MIS 3-2 transition (ca $29 \mathrm{ka}$ ) coincides with a last phase of a major fluvial incision (Busschers et al., 2007; Hijma et al., 2012).

The incision, erosion and redeposition of older units therein appear related to increasingly colder climate settings during the time leading to the LGM at ca $18 \mathrm{ka}$.

Busschers et al. (2007) invoke glacio-isostasy to explain and interpret the fluvial evolution of the Meuse-Rhine system. During the Weichselian, multiple large-scale ice-sheet extensions occurred in Britain and Scandinavia. The build-up of the ice-sheets in the Late Middle and Late Pleniglacial caused uplift of the foreland area around the ice margin (forebulge). An area of maximal uplift during the Late Pleniglacial glaciation was situated in the northern Netherlands (for further references see Busschers et al., 2007). The increased incisions and marked shift of channel belt activity is interpreted as reflecting a 
response to glacio-isostatic upwarping of the area. This induced a lateral shift of the river valley to the south because the north of the valley was updoming slightly more than south. Busschers (2008) also noted the fresh appearance of deposits reworked under these very cold conditions, as did Janssen (1965) earlier for Schelde deposits near Zelzate.

It is well possible that the interval in borehole Schelphoek previously interpreted as Eem Formation (Slupik et al., 2007) corresponds with one of the incisive episodes of the RhineMeuse (from MIS $5 a / 4-3 / 2$ ).

\section{Oosterschelde Gelasian mammals are mostly derived from a Late Pleistocene river lag}

The basal lag of the Koewacht Formation in the Moriaanshoofd Borehole contains a considerable concentration of bone material. It is most likely that the vertebrate remains dredged from the adjacent 0osterschelde channels at similar depth are derived from this basal lag. The basal lag material which we presume to be the source of the vertebrate remains has been reworked and concentrated partly from eroded upper parts of the Maassluis Formation (Slupik et al., 2007), and partly from reworked freshwater deposits of similar or later age. Some of the arvicolids in the Moriaanshoofd lag deposits can be assigned to biozone MNR1 of Tesakov (2004), age ca 2.1-2.25 Ma. In previous work, the larger vertebrates found in the 0osterschelde assemblage have been estimated at approximately 1.9 Ma (De Vos et al., 1995, 1998) on the basis of correlations with other (absolutely dated) European localities. This difference of 0.2 to $0.35 \mathrm{Ma}$ might indicate that the age estimates for the larger vertebrate faunas might require revision, but at the same time there is no reason why material dating from different ages should not have been incorporated in the lag deposits. It might also be that the eroded top part of the Maassluis Formation in the oosterschelde area contained sediments as young as ca 1.9 Ma. Local reworking of Middle Pleistocene material into the coarse lag deposits could explain the recovery of a large Trogontherium tibial fragment, suggesting a Middle Pleistocene age, from the deep channel of the Oosterschelde, close to the Moriaanshoofd Borehole (Mayhew et al., 2008).

\section{Extensive reworking challenges a straightforward lithostratigraphic subdivision}

The record of the Koewacht Formation indicates a southern origin of the material and fauna, and also locally reworked, possibly also fluvial, deposits. The Kreftenheye Formation shows another, Rhine, source. The record of the borehole shows Schelde-derived deposits as far north as Schouwen-Duiveland. Schelde-derived deposits in the southwest Netherlands have very recently been assigned to the Koewacht Formation - a lithostratigraphic unit defined by Kiden (2010). The Koewacht Formation consists of predominantly sandy deposits of rivers draining the Schelde basin in Belgium and northern France during the Saalian, Eemian and Weichselian through a wide paleovalley north of Ghent (Fig. 4), the so-called 'Flemish Valley' (Tavernier, 1946; Tavernier \& De Moor, 1974). The Kreftenheye Formation as defined by Buschers \& Weerts (2003) is a lithostratigraphic unit that consist of Rhine River deposits, with additional minor amounts of material from tributary rivers. Here we have not studied in depth the lithological composition of the fluvial units (and especially the gravel) in the Moriaanshoofd Borehole, so we cannot subdivide the deposits in more detail, but we have sufficient evidence of lithological differences (see above) to assign the deposits to the Koewacht Formation that consists of the fluvial units of a southerly origin and also abundant locally reworked (possibly also fluvial) deposits.

The very large role of reworking in the genesis of the stratigraphic successions in the southwestem Netherlands shows that a simple lithological-based (cf. IUGS approach) or a more complex facies-based approach (as advocated by the Dutch Geological Survey) is insufficient for a complete understanding of the geological units. The debate over lithology-based versus genetic-based stratigraphic concepts is very long-standing (Cox and Sumbler, 1999).

According to the code of stratigraphic nomenclature (Salvador, 1994), lithostratigraphic units should be defined and characterised on the basis of lithological properties of rocks bodies and their stratigraphic relations. We show that in the settings studied here some interpretation of the genesis of deposits is required in order to understand the lithological units.

In the Antwerp area massive reworking of mostly Pliocene strata into the Late Weichselian 'Schelde Sands' produced a lithological unit rich in fossils that barely differs from some of the locally underlying Pliocene units. Similarly, the base of the Maassluis Formation has only been understood in the nearby Schelphoek borehole (Slupik et al., 2007) through biostratigraphic and taphonomical evidence. The basal part there also is completely dominated by reworked Pliocene sediments and fossils giving it the appearance of the underlying 0osterhout Formation. So we argue for the involvement of facies successions (or even sequences) as a necessary step in defining geological formations in this region.

\section{Conclusions}

The Moriaanshoofd Borehole contains a stratigraphic record of Gelasian (Early Pleistocene) to Holocene deposits represented by six formations.

The Gelasian Maassluis Formation comprises recurring depositional facies reflecting cyclical deposition within a tidal setting. Between the top of the Maassluis Formation and the overlying deposits of Late Quaternary age a major hiatus exists comprising approximately $>2.2 \mathrm{Ma}$. 
During Weichselian times (33-24 ka), a proto-Schelde River shaped the northern Oosterschelde area. The river reworked substantial amounts of Early and Middle Pleistocene deposits. At the base of the Schelde derived fluvial sequence that we here attribute to the Koewacht Formation, Gelasian vertebrate faunas were concentrated. This channel lag is almost certainly the main source for the rich vertebrate faunas dredged from the adjacent Oosterschelde deep channel.

\section{Acknowledgements}

The authors would like to express their gratitude to Prof. Dr P. de Boer (Utrecht University) for stimulating discussion and constructive comments that resulted in significant improvement of the manuscript. Mr H. van Elburg drilled the borehole and Mr M. van den Bosch did the sampling and made the lithological descriptions, and Mr R. Last assisted during the boring. Mr C. Barnard (NCB Naturalis) assisted with sample processing. The management of the 'Waterschap Zeeuwse Eilanden' in Middelburg gave permission to drill the borehole and 'Staatsbosbeheer' provided access to the location. Mr J.J.P.M. Asselbergs - formerly mayor of Schouwen-Duiveland - supported us in many ways during preparatory procedures and during the drilling. Mr T. Meijer is thanked for valuable comments on the molluscan interpretations. Dr D. Nolf (KBIN, Brussels) provided us with distribution data of the Aalter Formation. Two reviewers: Dr K. Kasse and Dr F. Busschers significantly improved the manuscript after its first submission.

\section{References}

Bosch, J.H.A., 1999. Standaard Boor Beschrijvingsmethode, versie 5. Internal report TNO, rapport NITG 98-205-A, $88 \mathrm{pp.}$

Briant, R.M., Bateman, M.D., Coope, G.R. \& Gibbard, P.L., 2005. Climatic control on Quaternary fluvial sedimentology of a Fenland Basin river, England. Sedimentology 52: 1397-1423.

Busschers, F.S., 2008. Unravelling the Rhine. Response of a fluvial system to climate change, sea-level oscillation and glaciations. PhD thesis, Vrije Universiteit Amsterdam, 183 pp.

Busschers, F.S. \& Weerts, H.J.T., 2003. Formatie van Kreftenheye. In: Lithostratigrafische Nomenclator van de Ondiepe Ondergrond. Retrieved 2013 from www,dinoloket,nl/formatie-van-kreftenheye.

Busschers, F.S., Kasse, C., Van Balen, R.T., Vandenberghe, J., Cohen, K.M., Weerts, H.J.T., Wallinga, J., Johns, C., Cleveringa, P. \& Bunnik, F.P.M., 2007. Late Pleistocene evolution of the Rhine-Meuse system in the southern North Sea basin: imprints of climate change, sea-level oscillation and glacioisostacy. Quaternary Science Reviews 26: 3216-3248.

Cox, B.M. \& Sumbler, M.G., 1999. Lithostratigraphy: principles and practice. In: Doyle, P. \& Bennett, M.R. (eds): Unlocking stratigraphical record. Advances in modern stratigraphy. John Wiley \& Sons Ltd; 11-27.

Dalrymple, R.W., 1994. Tidal depositional systems, In: Walker, R.G. (ed.): Facies models: response to sea level change. Geological Association of Canada: 195-218.
De Bruyne, R.H., De Graaf, A. \& Hoeksema, D.F., 1987. Marine mollusks new for the Netherlands, washed ashore at the beaches of Ouddorp (GoereeOverflakkee, Province of Zuid-Holland), with some remarks on the occurrence of Alteneum dawsoni (Jeffreys, 1864). Basteria 51: 67-78.

De Mulder, E.F.J., Geluk, M.C., Ritsema, I., Westerhoff, W.E. \& Wong Th.E. (eds), 2003. De ondergrond van Nederland. Geologie van Nederland, deel 7. Nederlands Instituut voor Toegepaste Geowetenschappen TN0, $379 \mathrm{pp}$.

De Vos, J, Mol, D. \& Reumer, J.W.F., 1995. Early Pleistocene Cervidae (Mammalia, Artiodactyla) from the Oosterschelde (the Netherlands), with a revision of the cervid genus Eucladoceros Falconer, 1868. Deinsea 2: 95-121.

De Vos, J., Mol, D. \& Reumer, J.W.F., 1998. Early Pleistocene mammalian remains from the 0osterschelde or Eastern Scheldt (Province of Zeeland, the Netherlands). In: Van Kolfschaten T. \& Gibbard P.L. (eds): The dawn of the Quaternary. Proceedings of the SEQS-EuroMann symposium 1996. Mededelingen Nederlands Instituut voor Toegepaste Geowetenschappen TN0 60: 173-186.

Doppert, J.W.C., Ruegg, G.H.J., Van Staalduinen, C.J., Zagwijn, W.H. \& Zandstra, J.G., 1975. Lithostratigrafie. Formaties van het Kwartair en Boven-Tertiair in Nederland. In: Zagwijn, W. H. \& Van Staalduinen, C. J., (eds): Toelichting bij geologische overzichtskaarten van Nederland. Rijks Geologische Dienst (Haarlem): 11-56.

Du Four, L., Schelfaut, K., Vanheteren, S., Van Dijk, T. \& Van Lancker, V., 2006. Geologie en sedimentologie van het Westerscheldemondingsgebied. In: Coosen, J., Mees, J., Seys, J. \& Fockedey, N. (eds): Symposium: The Vlakte van de Raan van onder het stof gehaald. VLIZ Special Publication, 35 III. Vlaams Instituut voor de Zee (VLIZ) (Dostende), $135 \mathrm{pp}$.

Hijma, M.P., Cohen, K.M., Hoffmann, G., Van der Spek, A.J.F. \& Stouthamer, E., 2009. From river valley to estuary: the evolution of the Rhine mouth in the early to middle Holocene (western Netherlands, Rhine-Meuse delta). Netherlands Journal of Geosciences - Geologie en Mijnbouw 88(1): 13-53.

Hijma, M.P., Van der Spek, A.J.F. \& Van Heteren, S., 2010. Development of a midHolocene estuarine basin, Rhine-Meuse mouth area, offshore the Netherlands, Marine geology 271: 198-211.

Hijma, M.P. \& Cohen, K.M., 2010. Timing and magnitude of the sea-level jump preluding the 8200 yr event. Geology 38: 275-278.

Hijma, M.P. \& Cohen, K.M., 2011. Holocene transgression of the Rhine river mouth area, the Netherlands / Southern North Sea: palaeogeography and sequence stratigraphy. Sedimentology 58: 1453-1485.

Hijma, M.P., Cohen, K.M., Roebroeks, W., Westerhoff, W.E. \& Busschers, F.S., 2012. Pleistocene Rhine-Thames landscapes: geological background for hominin occupation of the southern North Sea Jegion. Journal of Quatemary Science 27(1): 17-39.

ICN/IUGS, 2009. Ratification of the definition of the base of Quaternary System/ Period (and top of the Neogene System/Period), and redefinition of the base of the Pleistocene Series/Epoch (and top of the Pliocene Series/Epoch). www.stratigraphy.org.

Janssen, A.W., 1965. Mollusca uit de pleistocene ontsluiting te Zelzate. Mededelingen van de Werkgroep Tertiaire en Kwartaire Geologie 2: 24-37.

Johnson, H.D. \& Baldwin, C.T. 1996. Shallow clastic seas. In: Reading, H.G. (ed.): Sedimentary environments: processes, facies and stratigraphy. Blackwell Publishing: 232-280.

Kiden, $\boldsymbol{P}_{\star}, 1991$. The Lateglacial and Holocene evolution of the Middle and Lower River Scheldt, Belgium. In: Starkel L., Gregary K.J. \& Thornes J.B., (eds): Temperate Palaeohydrology. John Wiley \& Sons: 283-299. 


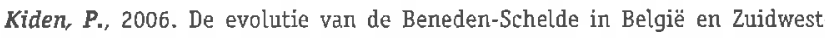
Nederland na de laatste ijstijd. Belgeo 2006/3: 279-294.

Kiden, $\boldsymbol{P}_{*}, 2010$. Formatie van Koewacht. In: Lithostratigrafische Nomenclator van de Ondiepe 0ndergrond. Retrieved 2013 from www.dinoloket.nl/ formatie-van-koewacht.

Mayhew, D.F. \& Gibbard, P.L., 1998. Early Pleistocene small mammal remains and pollen flora from the Crag at Weybourne, Norfolk, Fngland. In: Van Kolfschoten, T. \& Gibbard, P.L. (eds): The dawn of the Quatemary. Proceedings of the SEQS-EuroMam symposium 1996. Mededelingen Nederlands Instituut voor Toegepaste Geowetenschappen TNO 60: 263-269.

Mayhew, D.F. \& Stuart, A.J., 1986. Stratigraphic and Taxonomic revision of the fossil vole remains (Rodentia, Microtinae) from the Lower Pleistocene deposits of Eastern England. Philosophical Transactions of The Royal Society B 312 431-485.

Mayhew, D.F., De Vos, J. \& Van Veen, J.C., 2008. First record of Trogontherium cuvieri (Rodentia, Castoridae) from the 0osterschelde. Deinsea 12: 17-22.

Meijer, T. \& Cleveringa, P., 2009. Aminostratigraphy of Middle and Late Pleistocene deposits in the Netherlands and the southern part of the North Sea Basin. Global and Planetary Change 68: 326-345.

Nadeau, M.J., Grootes, P.M., Schleicher, M., Hasselberg, P., Rieck, A. \& Bitterling M., 1998. Sample throughput and data quality at the Leibniz-Labor AMS facility. Radiocarbon 40: 239-245.

Pevzner, M., Tesakov, A.S. \& Vangengeim, E., 1998. The position of the Tizdar locality (Taman peninsula, Russia) in the magnetochronological scale. Paludicola 2: 95-97.

Reading, H.G. \& Collinson, J.D., 1996. Clastic coasts, In: Reading, H.G. (ed.): Sedimentary environments: processes, facies and stratigraphy. Blackwell Publishing: 154-231.

Reumer, J.W.F, Van Veen, J.C., Van der Meulen, A.J., Hordijk, L.W. \& De Vos, J. 1998. The first find of small mammals (Desmaninae, Arvicolidae) from the Early Pleistocene Oosterschelde fauna in the Netherlands. Deinsea 4: 41-45.

Reumer, J.W.F., Mayhew, D.F. \& Van Veen, J.C., 2005. Small mammals from the Late Pliocene 0osterschelde dredgings. Deinsea 11: 103-118.

Salvador, A. (ed.), 1994. International stratigraphic guide: a guide to stratigraphic classification, teminology and procedure. International subcommission on stratigraphic classification of IUGS international commission on stratigraphy. Geological Society of America (Boulder), 214 pp.

Schokker, J., 2003. Patterns and processes in a Pleistocene fluvio-aeolian environment. Roer Valley Graben, south-eastern Netherlands. Netherlands Geographical Studies 314, 142 pp.

Schokker, J., De Lang, F.D., Weerts, H.J.T., Den Otter, C. \& Passchier, S., 2005 Formatie van Boxtel. In: Lithostratigrafische Nomenclator van de Ondiepe Ondergrond. Retrieved 2013 from www.dinoloket.nl/formatie-van-boxtel.

Schokker, J., Weerts, H.J.T., Westerhoff, W.E., Berendsen, H.J.A. \& Den Otter, C. 2007. Introduction of the Boxtel Formation and implications for the Quaternary lithostratigraphy of the Netherlands. Netherlands Journal of Geosciences - Geologie en Mijnbouw 86 (3): 197-210.

Slupik, A.A., Wesselingh, F.P., Janse, A.C. \& Reumer, J.W.F., 2007. The stratigraphy of the Neogene-Quaternary succession in the southwest Netherlands from the Schelphoek borehole (42G4-11/42G0022) - a sequencestratigraphic approach. Netherlands Journal of Geosciences - Geologie en Mijnbouw 86 (4): 317-332.
Spaink, G., 1975. Zonering van het mariene Onder-Pleistoceen en Plioceen op grond van mollusker fauna's. In: Zagwijn, W.H. \& Van Staalduinen, C.J., (eds): Toelichting bij geologische overzichtskaarten van Nederland. Rijks Geologische Dienst (Haarlem): 118-122.

Steurbaut E. \& Nolf, D., 1989. The stratotype of the Aalter sands (Eocene of SW Belgium): stratigraphy and calcareous nannoplankton. Mededelingen van de Werkgroep Tertiaire en Kwartaire Geologie 26 (1): 11-28.

Stuiver, M. \& Polach, H.A., 1977. Discussion: Reporting of ${ }^{14}$ C Data. Radiocarbon 19(3): 355-363.

Tavernier, R., 1946. L'évolution du Bas Escaut au Pléistocène supérieur. Bulletin de la Société Belge de Géologie 65: 106-125.

Tavernier, R. \& De Moor, G., 1974. L'évolution du Bassin de l'Escaut. In: Macar P. (ed.): L'évolution des bassins fluviaux de la Mer du Nord méridionale. Colloque du Centenaire de la Société Géologique de Belgique, Iiège: 159-231.

Tesakov, A.S., 1998. Voles of the Tegelen fauna. In: Van Kolfschoten, T. \& Gibbard, P.L. (eds): The dawn of the Quaternary. Proceedings of the SEQS-EuroMam symposium 1996. Mededelingen Nederlands Instituut voor Toegepaste Geowetenschappen TNO 60: 71-134.

Tesakov A.S., 2004. Biostratigrafiya srednego pliotsena - eopleistotsena vostochnoi Evropy (Biostratigraphy of the Middle Pliocere-Eopleistocene of Eastern Europe). Russian Academy of Sciences, Transactions of the Geological Institute 554 Nauka (Moscow), 247 pp. (In Russian with English summary).

TNO, 2013. Lithostratigrafische Nomenclator van de Ondiepe Ondergrond, versie 2013. Retrieved 2013 from www.dinoloket.nl/nomenclator-ondiep.

Van den Berg, J.H., Boersma, J.R. \& Van Gelder, A., 2007. Diagnostic sedimentary structures of the fluvial-tidal transition zone. Evidence from deposits of the Rhine and Meuse. Netherlands Journal of Geosciences - Geologie en Mijnbouw $86(3): 287-306$

Van Kolfschoten, T., 1988. The Pleistocene mammalian fauna from the Zuurland borehole. In: Van Kolschoten, T. \& De Boer, P.L. (eds): The Zuurland-2 borehole. Mededelingen van de Werkgroep voor Tertiaire en Kwartaire geologie / Contibutions to Tertiary and Quaternary geology 25 (1): 73-86.

Von Rummelen, F.F.F.E., 1965. Blad Zeeuwsch-Vlaanderen West en 0ost. Toelichtingen bij de Geologische Kaart van Nederland 1:50.000. Rijks Geologische Dienst (Haarlem), 79 pp.

Van Rummelen, F.F.F.E., 1970. Blad Schouwen-Duiveland. Toelichting bij de geologische kaart van Nederland 1:50.000. Rijks Geologische Dienst (Haarlem), $116 \mathrm{pp}$.

Van Rummelen, F.F.F.E., 1978. Blad Beveland. Toelichting bij de geologische kaart van Nederland 1:50.000. Rijks Geologische Dienst (Haarlem), 138 pp.

Van Strydonck, M. \& Van der Borg, K., 1990-1991. The Construction of a preparation line for AMS-targets at the Royal Institute for Cultural Heritage Brussels. Bulletin Kon. Inst. v. h. Kunstpatrimonium 23: 228-234.

Van Voorthuysen, J.H., 1957. Algemeen geologisch overzicht tot een diepte van 40 m. In: De Ridder N.A. (ed.): Agrohydrologische profielen van Zeeland. Ministerie van Landbouw, Staatsdrukkerij ('s-Gravenhage).

Vos, P.C., Bazelmans, J., Weerts, H.J.T. \& Van der Meulen, M.J. (eds.), 2011. Atlas van Nederland in het Holoceen (Amsterdam), $94 \mathrm{pp}$.

Weerts, H.J.T., 2003. Formatie van Naaldwijk. In: Lithostratigrafische Nomenclator van de Ondiepe Ondergrond. Retrieved 2013 from www.dinoloket.nl/ formatie-van-naaldwijk. 
Weerts, H.J.T., Cleveringa, P., Ebbing, J.H.J., De Lang, F.D. \& Westerhoff, W.E., 2000. De lithostratigrafische indeling van Nederland: Formaties uit het Tertiair en Kwartair. TNO-rapport NITG 00-95-A, TNO-NITG (Utrecht).

Weerts, H.J.T. \& Busschers, F.S., 2003. Formatie van Nieuwkoop. In: Lithostratigrafische Nomenclator van de Ondiepe Ondergrond. Retrieved 2013 from www.dinoloket.nl/formatie-van-nieuwkoop.

Westerhoff, W.E., Geluk, M.C. \& De Mulder, E.F.J., 2003. Geschiedenis van de ondergrond. In: De Mulder, E.E.J., Geluk, M.C., Ritsema. I., Westerhoff, W.E. \& Wong. T. (eds): De ondergrond van Nederland. Nederlands Instituut voor Toegepaste Geowetenschappen TNO: 119-246. 


\section{Appendix 1 - Lithological description of the Moriaanshoofd borehole}

Depth is given in metres below surface (= $4.19 \mathrm{~m}$ above mean sea level - N.A.P.). MSMoA, MSMoA, MSMoB, MSMoC - facies in the Maassluis Formation; KWMoA, KWMoB - facies in the Koewacht Formation; KR - Kreftenheye Formation; BX - Boxtel Formation; NAMoA, NAMoB, NAMoC - facies in the Naaldwijk Formation; NI - Nieuwkoop Formation.

\begin{tabular}{|c|c|c|}
\hline $\begin{array}{l}\text { Depth } \\
\text { (m b.s.) }\end{array}$ & Sediment features & $\begin{array}{l}\text { Facies/ } \\
\text { units }\end{array}$ \\
\hline $0-0.5$ & Black soil; slightly silty and fine sandy; highly calcareous & \\
\hline $0.5-6$ & Grey sand; anthropogenic & \\
\hline $6-7$ & $\begin{array}{l}\text { Grey sand; fine to medium fine with numerous very thin clay layers; numerous shell fragments; strongly calcareous; } \\
\text { bottom part consists of finely layered sandy-clayey complex with some shell fragments; }\end{array}$ & NAMOA \\
\hline $7-7.75$ & Dark grey clay; silty; with fine to medium fine sand partings; some shell fragments; highly calcareous & \\
\hline $7.75-8$ & Black peat with wood remains & NI \\
\hline $8-8.5$ & Grey clay; weakly silty; rather firm; peaty towards the top; strongly calcareous & NAMoB \\
\hline $8.5-12$ & $\begin{array}{l}\text { Grey clay-sandy finely layered complex; clay is slightly silty; sand is fine; few shell fragments; some wood pieces in } \\
\text { the uppermost part; strongly calcareous }\end{array}$ & \\
\hline $12-14$ & $\begin{array}{l}\text { Grey clay; rather silty; rather firm with layers of fine sand; some shell fragments; strongly calcareous; lowermost } \\
\text { part contains finely layered clayey-sandy complex with some shell fragments }\end{array}$ & \\
\hline 14-16 & Grey sand; fine to medium fine; with thin clay layers; some fine shell fragments in the bottom part; strongly calcareous & NAMOC \\
\hline $16-18$ & Grey to dark grey clay-sandy layered complex; clay is silty; sand is medium fine; strongly calcareous & \\
\hline $18-20.5$ & Grey to dark grey clay; silty to fine sandy; few shell fragments in the bottom part; strongly calcareous & \\
\hline $20.5-21.7$ & Grey sand; medium fine to medium coarse; with a few clay layers; some fine shell fragments; strongly calcareous & \\
\hline $21.70-22$ & Dark brown peat; firm & NI \\
\hline $22-23.5$ & Light grey sand; medium coarse; not calcareous & BX \\
\hline $23.5-28$ & Light grey sand; medium fine to coarse; not calcareous & \\
\hline $28-29$ & Light grey sand; medium coarse; trace of shell fragments; slightly calcareous & $\mathrm{KR}$ \\
\hline
\end{tabular}

29-31 Grey sand; coarse; some clay clasts in the bottom part; some fine gravel; rather abundant shells and shell fragments in the upper part and some fine shell fragments in the lower part; some wood fragments; slightly calcareous

31-32.5 Grey sand; medium coarse; rather calcareous

32.5-34.5 Grey sand; medium coarse to coarse; some shell fragments in the upper part and rather abundant shells; shell fragments and pieces of reworked peat in the lower part; weakly calcareous

34.5-36 Grey sand; medium coarse to coarse; some clay clasts in the lowermost part; abundant shells and shell fragments; few wood fragments and reworked peat; not calcareous

36-36.5 Darkish grey sand; fine to medium fine; with a few light grey hard clay clasts; some black clasts; not calcareous Brown claystone clasts; rolled; up to a few centimetres in size

36.5-37 Dark grey sand; fine to medium fine; strongly silty; some mica; some light grey consolidated clay clasts; some black lithic fragments; not calcareous

37-40 Dark grey sand; fine to medium fine; thin; containing silt to rather strongly silty; fine sandy clay layers; some mica; $\mathrm{MSMOB}$ some light grey consolidated clay clasts

40-41 Dark grey sand; fine to medium fine; silty; thin; fine sandy clay layers; some light grey consolidated clay clasts; some black lithic clasts; some mica; not calcareous

41-43 Dark grey sand; medium fine; a few fine clay clasts; not calcareous;

43-44 Dark grey sand; fine to medium fine; some very thin clayey layers; some fine clay clasts; abundant mica; not calcareous

44-45 Dark grey sand; fine to medium fine; rather strongly silty; numerous thin clay layers; some clay pebbles; not calcareous Dark grey sand; fine to medium fine; in the lower part fine to medium coarse; scarce clay fragments; some mica; not calcareous

47-48 Dark grey sand; a few thin; silty clay layers; single clay piece; some mica; not calcareous

48-49 Grey sand; medium fine to medium coarse; scarce clay clasts in the lower part; a few wood fragments in the uppermost part; not calc.

49-53 Grey sand; medium tine; some mica; not calcareous

53-54 Grey sand; madium fine to medium coarse; not calcareous Grey sand; medium fine to medium coarse; some clay pieces; some shell fragments; a few fragments of wood; slightly calcareous Grey sand; medium coarse to coarse; silty; some clay pieces; some wood fragments; slightly calcareous Dark grey sand; medium coarse to coarse; very abundant shell fragmentsand shell gravel; a few flat clay pebbles; slightly calcareous 